\title{
The Efficacy and Safety of Integrated Chinese Plus Western Medicine Compared with Western Medicine Alone in the Treatment for Purpuric Nephritis: A Meta-Aalysis
}

\author{
Changchun Shi, Meng Peng*, Yingying Du, Wenxing Li, Yonghan Hu, Tingting Wu \\ Department of Health Management, Medical College of Hangzhou Normal University, Hangzhou city, China \\ Email address: \\ shicc1961@163.com@MengPeng.com (Changchun Shi),805297783@qq.com@MengPeng.com (Meng Peng), \\ fancy1005@126.com@MengPeng.com (Yingying Du),971074145@qq.com@MengPeng.com (Wenxing Li), \\ 704059055@qq.com@MengPeng.com (Yonghan Hu),577136497@qq.com@MengPeng.com (Tingting Wu) \\ ${ }^{*}$ Corresponding author
}

\section{To cite this article:}

Changchun Shi, Meng Peng, Yingying Du, Wenxing Li, Yonghan Hu, Tingting Wu. The Efficacy and Safety of Integrated Chinese Plus Western Medicine Compared with Western Medicine Alone in the Treatment for Purpuric Nephritis: A Meta-Aalysis. Science Journal of Public Health. Vol. 8, No. 2, 2020, pp. 19-35. doi: 10.11648/j.sjph.20200802.11

Received: January 9, 2020; Accepted: January 27, 2020; Published: March 10, 2020

\begin{abstract}
Objective: This research is aimed to systematically evaluate the efficacy and safety of Chinese plus Western Medicine treatment for Childhood purpuric nephritis by Meta-analysis, in order to improve the cure rate and reduce adverse reactions of this disease, then provide guidance for clinical medication. Methods: There are six major databases including CNKI, Pubmed, Embase, World Wide Database, Web of science and Science Direct retrieved through the Internet, and the randomized-controlled trials of the combined therapy for Childhood purpuric nephritis published both in Chinese and English from the 1999 to October 2019 were collected. Meta-analysis by R software 3.5.0 was adopted to analyze the effective index and adverse effect. Results: In total 92 papers of randomized-controlled clinical studies were enrolled. HSPN children treated with combined therapy demonstrated a significant increase in total effective rate $(\mathrm{RR}=1.19 ; 95 \% \mathrm{CI}(1.16-1.21), \mathrm{P}<0.001)$ when compared with Western medicine alone. Our study also found that Chinese plus Western medicine had an advantage on decreasing 24-hour urine protein (WMD=-0.47; 95\%CI $(-0.53,-0.41) ; \mathrm{p}<0.001)$ and urine erythrocyte count (WMD=-8.88; $95 \%$ CI $(-9.80,-7.96) ; \mathrm{p}<0.001)$ when compared with Western medicine. Children seemed to benefit more from combined treatment in the adverse effect $(\mathrm{RR}=0.64 ; 95 \% \mathrm{CI}(0.46,0.87) ; \mathrm{p}<0.01)$. Conclusions: Integrated Chinese plus Western medicine treatment was effective for Childhood purpuric nephritis, which may be a superior alternative for HSPN. Nevertheless, long-term, high-quality and multicenter RCTs are required to make the results more convincing.
\end{abstract}

Keywords: Chinese Plus Western Medicine, Childhood Purpuric Nephritis, Efficacy and Safety, Randomized-Controlled Trials, Meta-Analysis

\section{Introduction}

Henoch-Schonlein purpura nephritis (HSPN) is a disease of kidney which damage the kidney within 6 months after the onset of Henoch-Schonlein purpura (HSP) [1]. At present, the specific pathogenesis of HSPN is not completely identified. It is generally accepted that some virus, bacteria, specific substances and other factors lead to allergic reactions, the disorder of cellular and humoral immunity. Eventually, the IgA immune complex deposits in the glomerulus can be seriously damaged. As a result, those substance damage the kidney $[2,3]$. There are also domestic studies which point out that the disorder of intestinal microflora in children is also related to the pathogenesis of this disease [4]. Most children with HSPN have a good prognosis. However, the report demonstrated that around $15 \%$ to $20 \%$ of children will eventually develop chronic renal failure. Thus, it is of great importance to pay enough attention to it. There is no unified treatment plan for this disease because of the diverse clinical manifestations and complex pathological classification of HSPN. At present, the treatment mainly includes symptomatic treatment, immunosuppressant and 
glucocorticoid application $[5,6]$. In recent years, more and more papers revealed that the integrated treatment of children HSPN with traditional Chinese and Western medicine has achieved excellent result. They determined the treatment program of Western medicine according to the clinical manifestation and pathological classification of children. At the same time, the individualized dialectical treatment combined with Chinese medicine classification. Many academic reports showed that the traditional Chinese medicine have the function of synergistic detoxification for glucocorticoid and it can be quite favorable [7, 8]. In this paper, a meta-analysis is conducted on the randomized controlled trials (RCT) of the combined treatment for children HSPN with traditional Chinese and Western medicine. The aim of the research is to objectively evaluate the effectiveness and possible advantages of the treatment so as to provide a basis for the guidance of rational clinical medication.

\section{Materials and Methods}

\subsection{Search Strategy}

The research articles were searched on the CNKI, Pubmed, Embase, World Wide Database, Web of science and Science Direct databases (1999-October 2019; key words: "purpura nephritis" and" integrated Traditional/Chinese and/plus Western Medicine" or" combined Chinese and Western Medicine). The academic materials was limited to the published studies that focused on RCT or clinical trials of integrated Chinese and Western Medicine therapy for Childhood/children HSPN. The publications are in both English-language and Chinese-language. In order to ensure complete literature retrieval, Chinese and English keywords were used to carry out secondary search on traditional Chinese medicine, Chinese and western medicine, integrated Chinese and western medicine. Meanwhile, retrospective research was conducted on the related literature references.

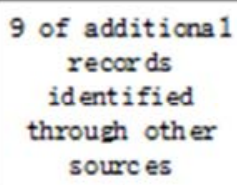

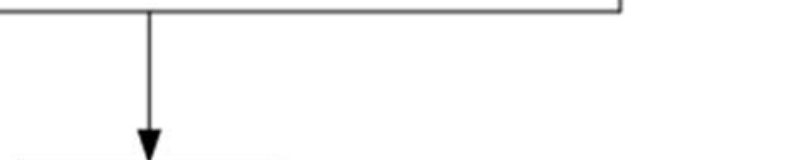

2405 of records excluded

(1) Reviem $n=133$

(2) Conference

summary $n=24$

(3) 2248 of

records after

dupli cat es

removed

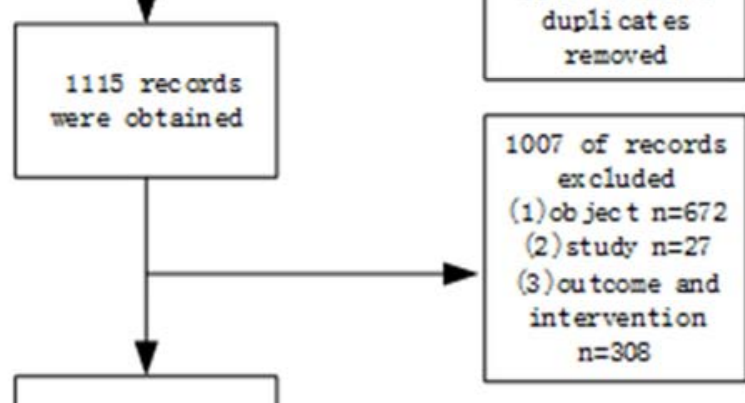

108 records

were obtained

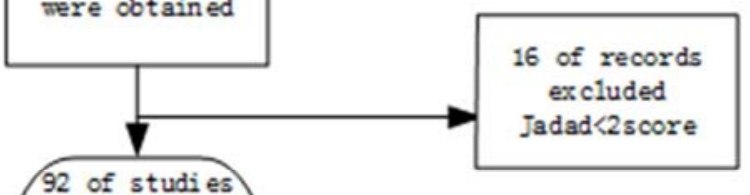

92 of studies
included in
quantitative
synthesis
(cum lat ive
peta-analysis)

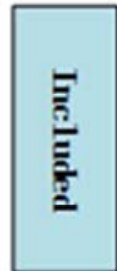

Figure 1. Flowchart illustrating the selection of studies. 


\subsection{Inclusion and Exclusion Criteria}

The inclusion and exclusion criteria were formulated according to the PICOS features which were included in the RCT: P (patient, research object), I (intervention method), C (control, comparative intervention method), O (outcomes, measurement outcomes), $\mathrm{S}$ (studies, research design).

The inclusion criteria were as follows. (a) P: children with HSPN and all met the diagnostic criteria of the evidence-based guidelines for the diagnosis and treatment of HSPN in the kidney disease group of the Chinese Medical Association in 2009 [9] or in 2000. (b) I: Chinese medicine (self-designed prescription, traditional medicine) plus hormones and/or immunosuppressants. TCM compounds include traditional decoction, tablets made with modern technology, injection, capsule. Administration may include oral, intravenous, enema, or combination therapy. Chinese medicine compound refers to a group of drugs which are made up of appropriate dosage and proper compatibility according to the composition principle after the treatment is decided by the reason of syndrome differentiation. (c) C: 24-hour urine protein ration $>250 \mathrm{mg}$, need to be treated with hormone and/or immunosuppressant. (d) O: The outcome measures should be included total effective rate and/or 24-hour urine protein ration and/or Urine erythrocyte count, the safety index was side-effect. (e) S: Randomized controlled trial (random number table, computer random arrangement, coin toss, lottery, etc.). Semi-randomized controlled trial (grouped by visit order, parity of birth date, etc.). (f) Basic treatment: All groups should include basic treatment, such as, look for allergens and avoid allergenic foods and medications. Rest and keep warm. Give antihistamines to fight allergies. Take vitamin $\mathrm{C}$ and vitamin $\mathrm{P}$ to improve the fragility of capillary wall, hemostasis, anti-infection and other symptomatic treatment.

Following are the exclusion criteria. (a) No parallel or animal studies. (b) Patients have serious complication, infection and other acute and chronic serious heart and lung disease. (c) The control group also used Chinese medicine decoction, compound Chinese patent medicine, single Chinese medicine and so on. (d) The observation indicators did not include clinical total effective rate/ 24-hour urine protein quantitative / Urine erythrocyte count and other indicators reflecting the efficacy. (e) Retrospective study, experience summary, case report, etc. (f) Summary and only abstract without full text and data.

\subsection{Study Selection and Data Extraction}

A unified table was developed by two evaluators to extract data from the included literature, based on the inclusive and exclusive criteria through titles and abstracts. The parts expressed in tabular from were the first author, publication year, study design, demographic characteristics, number and intervention measures of patients enrolled in trial and control group, duration of intervention and follow-up, main outcome measures (the total effective rate/24-hour urine protein
ration/Urine erythrocyte count), side-effects. The consensus was reached by consulting the third investigator when disagreements happened.

\subsection{Outcome Measurements}

To evaluate the efficacy and safety of Chinese plus Western medicine, the ratio of recovery significant effect, effective rate is called total effective rate.

(1) Recovery: clinical symptoms and signs decreased $\geq 90 \%$, urine sediment erythrocyte and/or $24 \mathrm{~h}$ urine protein quantitative normal.

Significant effect: clinical symptoms and signs were reduced by $75 \% \sim 90 \%$, urine sediment erythrocyte decreased by $\geq 3 / \mathrm{HP}$ and/or 24 -hour urine protein ration decreased by $\geq 50 \%$.

Effective: clinical symptoms and signs decreased in 50\% $75 \%$, urine sediment erythrocyte decreased $\geq 2 / \mathrm{HP}$ and/or 24-hour urine protein ration decreased by $25 \%$ to $50 \%$. Different studies had their own definition of complete and partial remission, but all of them used the reduction of urinary protein as the indicator.

(2) 24-hour urine protein ration and Urine erythrocyte count.

(3) The safety index were the incidence of adverse reactions during treatment and follow-up.

\subsection{Quality Assessment and Data Synthesis}

Since the selected articles were all involved Chinese medicine, the JADAD Scale, which considered the randomization, blinding, follow-up, was used to assess the quality of studies that was scored from 0 to 5 by two investigators. At the same time, the Distribution of hidden is evaluated. 0-2 was classified as low quality, 3-5 were classified as high quality studies. All scores were over 2, suggesting that their outcomes were convictive.

Risk ratios (RR) with its $95 \%$ confidence interval (CI) were analyzed for dichotomous outcomes, while the mean difference (MD) was chosen for continuous variables, described with a $95 \%$ CI. $\mathrm{R}$ version 3.5.0 was used to conduct meta-analysis. We applied a random-effect model or a fixed-effect analysis depending on $\mathrm{I}^{2}$ and $\mathrm{P}$ which was to measure the heterogeneity. The effective and adverse rate are combined and tested with the escalc and rma. uni functions of the $\mathrm{R}$ metafor. 24-hour urine protein ration and Urine erythrocyte count mean with the metacont function of the $\mathrm{R}$ metafor. If the results show a statistical difference, the potential publication bias will be analyzed by "funnel plot". Funnel plot are tested by regtest function. If the graph is symmetrical, there will be no effect of publication bias. If the graph is asymmetric, there is publication bias. At last, mixed effect models were used to analyze heterogeneity in studies with high heterogeneity. Studies with high heterogeneity were analyzed for heterogeneity. 


\section{Results}

\subsection{Basic Characteristics of Included Studies}

A total of 7,394 children in the 92 studies were included in the systematic evaluation, all of whom were aged from 2 to 15 years old. All studies described and compared the baseline conditions of children in the experimental group and the control group in terms of age, sex ratio, disease course, with statistical significance between the two groups $(\mathrm{P}>0.05)$. On the whole, the study showed good inter-group equilibrium and comparability $(\mathrm{P}>0.05)$. The experimental group of 44 studies was treated with proprietary Chinese medicine capsules, tablets and injections, and the remaining 48 studies were treated with traditional Chinese medicine decoction. Western medicine in the control group was treated with glucocorticoids, including 11 studies that added CTX and other immunosuppressor, with the basic treatment measures in all studies were basically the same.

Table 1. Basic characteristics of included studies.

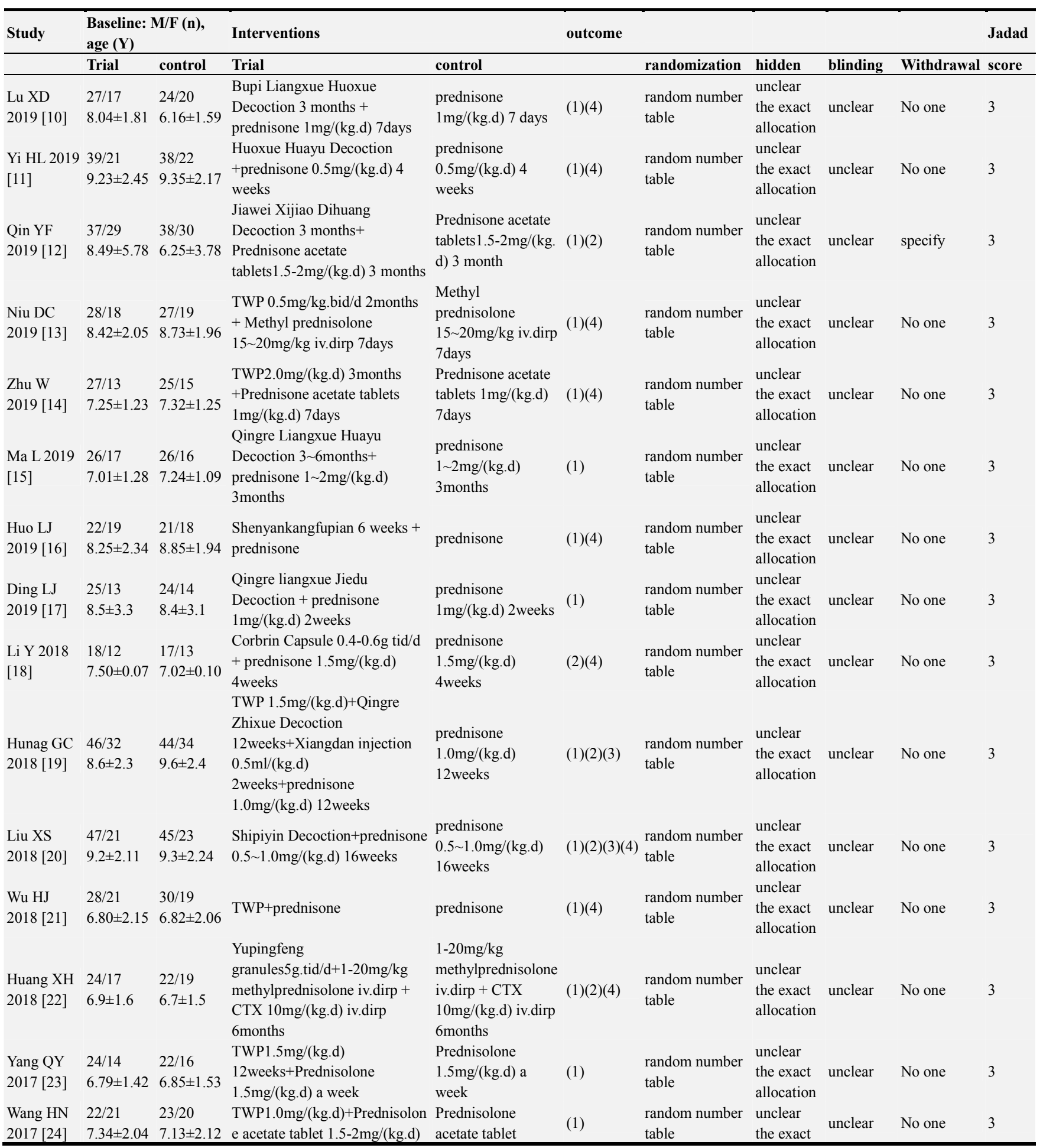




\begin{tabular}{|c|c|c|c|c|c|c|c|c|c|c|}
\hline Study & $\begin{array}{l}\text { Baseline: } 1 \\
\text { age }(Y)\end{array}$ & $\mathbf{M} / \mathbf{F}(\mathbf{n})$ & Interventions & & outcome & & & & & Jadad \\
\hline & Trial & control & Trial & control & & randomization & hidden & blinding & Withdrawal & score \\
\hline & & & 12weeks & $1.5-2 \mathrm{mg} /(\mathrm{kg} . \mathrm{d})$ & & & allocation & & & \\
\hline $\begin{array}{l}\text { Zhang L } \\
2017 \text { [25] }\end{array}$ & $\begin{array}{l}20 / 15 \\
6.92 \pm 1.48\end{array}$ & $\begin{array}{l}19 / 16 \\
7.08 \pm 1.54\end{array}$ & $\begin{array}{l}\text { TWP1.0mg/(kg.d)+Prednisolon } \\
\text { e } 1.5-2 \mathrm{mg} /(\mathrm{kg} . \mathrm{d}) 8 \text { weeks }\end{array}$ & $\begin{array}{l}12 \text { weeks } \\
\text { Prednisolone } \\
1.5-2 \mathrm{mg} /(\mathrm{kg} . \mathrm{d}) \\
8 \text { weeks }\end{array}$ & (1) & $\begin{array}{l}\text { random number } \\
\text { table }\end{array}$ & $\begin{array}{l}\text { unclear } \\
\text { the exact } \\
\text { allocation }\end{array}$ & unclear & No one & 3 \\
\hline $\begin{array}{l}\text { Liu XG } \\
2016[27]\end{array}$ & $\begin{array}{l}18 / 14 \\
8.03 \pm 4.29\end{array}$ & $\begin{array}{l}17 / 15 \\
7.98 \pm 3.60\end{array}$ & $\begin{array}{l}\text { Corbrin Capsule } 0.4-0.6 \mathrm{~g} \text { tid } / \mathrm{d} \\
+ \text { prednisone } 1.5 \mathrm{mg} /(\mathrm{kg} . \mathrm{d}) \\
\text { 4weeks }\end{array}$ & $\begin{array}{l}\text { prednisone } \\
1.5 \mathrm{mg} /(\mathrm{kg} . \mathrm{d}) \\
4 \text { weeks }\end{array}$ & (2) & $\begin{array}{l}\text { random number } \\
\text { table }\end{array}$ & $\begin{array}{l}\text { unclear } \\
\text { the exact } \\
\text { allocation }\end{array}$ & unclear & No one & 3 \\
\hline $\begin{array}{l}\text { Li J } 2016 \\
{[28]}\end{array}$ & $\begin{array}{l}18 / 14 \\
7.7 \pm 1.3\end{array}$ & $\begin{array}{l}19 / 12 \\
8.3 \pm 2.1\end{array}$ & $\begin{array}{l}\text { TWP1.0 1.5mg/(kg.d)+prednis } \\
\text { one } 12 \text { weeks }\end{array}$ & $\begin{array}{l}\text { prednisone } \\
12 \text { weeks }\end{array}$ & $(1)(4)$ & $\begin{array}{l}\text { random number } \\
\text { table }\end{array}$ & $\begin{array}{l}\text { unclear } \\
\text { the exact } \\
\text { allocation }\end{array}$ & unclear & No one & 3 \\
\hline $\begin{array}{l}\text { Sun ZH } \\
2016[29]\end{array}$ & $\begin{array}{l}16 / 20 \\
9.1 \pm 3.8\end{array}$ & $\begin{array}{l}17 / 19 \\
9.2 \pm 3.7\end{array}$ & $\begin{array}{l}\text { Fushen Decoction }+ \text { prednisone } \\
0.5 \mathrm{mg} \sim 2 \mathrm{mg} /(\text { kg.d) } 2 \text { months }\end{array}$ & $\begin{array}{l}\text { prednisone } \\
0.5 \mathrm{mg} 2 \mathrm{mg} /(\mathrm{kg} . \mathrm{d}) \\
2 \mathrm{months}\end{array}$ & $(1)(2)$ & $\begin{array}{l}\text { random number } \\
\text { table }\end{array}$ & $\begin{array}{l}\text { unclear } \\
\text { the exact } \\
\text { allocation }\end{array}$ & unclear & No one & 3 \\
\hline $\begin{array}{l}\text { Zhao QW } \\
2016[30]\end{array}$ & \multicolumn{2}{|c|}{$62 / 286.8 \pm 0.4$} & $\begin{array}{l}\text { Abelmosk capsule } \\
\text { tid/d+prednisone+CTX } 8 \text { weeks }\end{array}$ & $\begin{array}{l}\text { prednisone+CTX } 8 \\
\text { weeks }\end{array}$ & (1) & $\begin{array}{l}\text { random number } \\
\text { table }\end{array}$ & $\begin{array}{l}\text { unclear } \\
\text { the exact } \\
\text { allocation }\end{array}$ & unclear & No one & 3 \\
\hline $\begin{array}{l}\text { He JM } \\
2016[31]\end{array}$ & $\begin{array}{l}21 / 15 \\
7.2 \pm 2.1\end{array}$ & $\begin{array}{l}19 / 18 \\
7.6 \pm 2.6\end{array}$ & $\begin{array}{l}\text { Liangxue Shenyu Decoction } \\
\text { p.o+Prednisolone } 30 \mathrm{mg} /(\mathrm{kg} . \mathrm{d}) \\
\text { iv.dirp 3weeks }\end{array}$ & $\begin{array}{l}\text { Prednisolone } \\
\text { 30mg/(kg.d) iv.dirp } \\
\text { 3weeks }\end{array}$ & $(1)(2)(3)$ & $\begin{array}{l}\text { random number } \\
\text { table }\end{array}$ & $\begin{array}{l}\text { unclear } \\
\text { the exact } \\
\text { allocation }\end{array}$ & unclear & No one & 3 \\
\hline $\begin{array}{l}\text { Gu MM } \\
2016[32]\end{array}$ & $\begin{array}{l}19 / 15 \\
7.15 \pm 1.86\end{array}$ & $\begin{array}{l}18 / 16 \\
7.24 \pm 1.92\end{array}$ & $\begin{array}{l}\text { Liangxue Xiaoban granules } 4 \\
\text { weeks+ Prednisolone }\end{array}$ & Prednisolone & $(1)(4)$ & $\begin{array}{l}\text { random number } \\
\text { table }\end{array}$ & $\begin{array}{l}\text { unclear } \\
\text { the exact } \\
\text { allocation }\end{array}$ & unclear & No one & 3 \\
\hline $\begin{array}{l}\text { Liu XY } \\
2016[33]\end{array}$ & \multicolumn{2}{|c|}{$23 / 276.5 \pm 2.4$} & $\begin{array}{l}\text { TWP } 1.0 \mathrm{mg} /(\mathrm{kg} . \mathrm{d})+\text { Prednisolon } \\
\text { e } 3 \text { months }\end{array}$ & $\begin{array}{l}\text { Prednisolone } 3 \\
\text { months }\end{array}$ & $(1)(4)$ & $\begin{array}{l}\text { random number } \\
\text { table }\end{array}$ & $\begin{array}{l}\text { unclear } \\
\text { the exact } \\
\text { allocation }\end{array}$ & unclear & No one & 3 \\
\hline $\begin{array}{l}\text { Yin HW } \\
2016[34]\end{array}$ & \multicolumn{2}{|c|}{$35 / 396.2 \pm 1.1$} & $\begin{array}{l}\text { TWP1.0mg/(kg.d)+Prednisolon } \\
\text { e } 0.5-2 \mathrm{mg} /(\mathrm{kg} . \mathrm{d}) 3 \text { months }\end{array}$ & $\begin{array}{l}\text { Prednisolone } \\
0.5-2 \mathrm{mg} /(\mathrm{kg} . \mathrm{d}) \\
3 \text { months }\end{array}$ & $(1)(4)$ & $\begin{array}{l}\text { random number } \\
\text { table }\end{array}$ & $\begin{array}{l}\text { unclear } \\
\text { the exact } \\
\text { allocation }\end{array}$ & unclear & No one & 3 \\
\hline $\begin{array}{l}\text { Zhang XP } \\
2016[35]\end{array}$ & $\begin{array}{l}18 / 12 \\
7.8 \pm 3.32\end{array}$ & $\begin{array}{l}16 / 14 \\
8.19 \pm 3.36\end{array}$ & $\begin{array}{l}\text { Xiaodian } \\
\text { Decoction+Prednisolone } \\
0.5-1.0 \mathrm{mg} /(\mathrm{kg} . \mathrm{d}) 6 \text { weeks }\end{array}$ & $\begin{array}{l}\text { Prednisolone } \\
0.5-1.0 \mathrm{mg} /(\mathrm{kg} . \mathrm{d}) \\
\text { 6weeks }\end{array}$ & $(1)(2)(3)(4)$ & $\begin{array}{l}\text { random number } \\
\text { table }\end{array}$ & $\begin{array}{l}\text { unclear } \\
\text { the exact } \\
\text { allocation }\end{array}$ & unclear & No one & 3 \\
\hline $\begin{array}{l}\text { Liu FJ } \\
2015[36]\end{array}$ & $\begin{array}{l}68 / 40 \\
10.1 \pm 3.5\end{array}$ & $\begin{array}{l}44 / 40 \\
10.3 \pm 3.7\end{array}$ & $\begin{array}{l}\text { Chinese herbs }+ \text { prednisone } \\
0.4-1 \mathrm{mg} /(\mathrm{kg} . \mathrm{d}) 10 \text { weeks }\end{array}$ & $\begin{array}{l}\text { prednisone } \\
0.4-1 \mathrm{mg} /(\mathrm{kg} . \mathrm{d}) 10 \\
\text { weeks }\end{array}$ & $(1)(2)(4)$ & $\begin{array}{l}\text { random number } \\
\text { table }\end{array}$ & $\begin{array}{l}\text { unclear } \\
\text { the exact } \\
\text { allocation }\end{array}$ & unclear & No one & 3 \\
\hline $\begin{array}{l}\text { Lu W } 2015 \\
{[37]}\end{array}$ & $\begin{array}{l}12 / 8 \\
8.71 \pm 5.02\end{array}$ & $\begin{array}{l}9 / 11 \\
8.12 \pm 4.35\end{array}$ & $\begin{array}{l}\text { Huaiqihaung } \\
\text { granules+prednisone } 1 \text { month }\end{array}$ & prednisone 1 month & $(1)(4)$ & $\begin{array}{l}\text { random number } \\
\text { table }\end{array}$ & $\begin{array}{l}\text { unclear } \\
\text { the exact } \\
\text { allocation }\end{array}$ & unclear & No one & 3 \\
\hline \multirow[t]{2}{*}{$\begin{array}{l}\text { §Zhang SS } \\
2015[38]\end{array}$} & $26 / 149.2$ & $24 / 169.0$ & $\begin{array}{l}\text { TWP1.0mg/(kg.d)+prednisone } \\
0.5-1 \mathrm{mg} /(\mathrm{kg} . \mathrm{d}) 3 \text { months }\end{array}$ & $\begin{array}{l}\text { prednisone } \\
0.5-1 \mathrm{mg} /(\mathrm{kg} \cdot \mathrm{d}) \\
\text { 3months }\end{array}$ & $(1)(3)$ & $\begin{array}{l}\text { random number } \\
\text { table }\end{array}$ & $\begin{array}{l}\text { unclear } \\
\text { the exact } \\
\text { allocation }\end{array}$ & unclear & No one & 3 \\
\hline & 27/139.7 & & $\begin{array}{l}\text { TWP } 1.0 \mathrm{mg} /(\mathrm{kg} . \mathrm{d})+\text { Chinese } \\
\text { herbs } 3 \text { months }\end{array}$ & & & & & & & \\
\hline $\begin{array}{l}\text { Sun ZM } \\
2015 \text { [39] }\end{array}$ & $\begin{array}{l}25 / 17 \\
8.6 \pm 2.4\end{array}$ & $\begin{array}{l}26 / 15 \\
8.5 \pm 2.2\end{array}$ & $\begin{array}{l}\text { TWP1.0mg/(kg.d)+prednisone } \\
\text { 3months }\end{array}$ & prednisone 3 months & $(1)(2)(3)(4)$ & $\begin{array}{l}\text { random number } \\
\text { table }\end{array}$ & $\begin{array}{l}\text { unclear } \\
\text { the exact } \\
\text { allocation }\end{array}$ & unclear & No one & 3 \\
\hline $\begin{array}{l}\text { Zhao F } \\
2015 \text { [40] }\end{array}$ & $\begin{array}{l}21 / 19 \\
5.2 \pm 3.1\end{array}$ & $\begin{array}{l}23 / 17 \\
6.2 \pm 2.1\end{array}$ & $\begin{array}{l}\text { TWP } 1.0 \mathrm{mg} /(\mathrm{kg} . \mathrm{d})+\text { prednisone } \\
1.5-2.0 \mathrm{mg} /(\mathrm{kg} . \mathrm{d}) 3 \text { months }\end{array}$ & $\begin{array}{l}\text { prednisone } \\
1.5-2.0 \mathrm{mg} /(\mathrm{kg} . \mathrm{d}) \\
3 \text { months }\end{array}$ & (1) & $\begin{array}{l}\text { random number } \\
\text { table }\end{array}$ & $\begin{array}{l}\text { unclear } \\
\text { the exact } \\
\text { allocation }\end{array}$ & unclear & No one & 3 \\
\hline $\begin{array}{l}\text { Liu BZ } \\
2015[41]\end{array}$ & $\begin{array}{l}18 / 22 \\
8.1 \pm 2.5\end{array}$ & $\begin{array}{l}16 / 24 \\
7.8 \pm 3.2\end{array}$ & $\begin{array}{l}\text { Taohong Siwu Decoction } \\
\text { methylprednisolone } \\
1.5 \mathrm{mg} /(\mathrm{kg} . \mathrm{d}) 2 \text { weeks }\end{array}$ & $\begin{array}{l}\text { methylprednisolone } \\
1.5 \mathrm{mg} /(\mathrm{kg} . \mathrm{d}) \\
2 \text { weeks }\end{array}$ & (1) & $\begin{array}{l}\text { random number } \\
\text { table }\end{array}$ & $\begin{array}{l}\text { unclear } \\
\text { the exact } \\
\text { allocation }\end{array}$ & unclear & No one & 3 \\
\hline $\begin{array}{l}\text { Wu HM } \\
2015[42]\end{array}$ & \multicolumn{2}{|l|}{$60 / 368.2$} & $\begin{array}{l}\text { Qiong Danshen injection } \\
\text { triazine } \\
2.5 \sim 5 \mathrm{ml} / \mathrm{d}+\text { prednisolone } \\
\text { acetate } 1.5-2.0 \mathrm{mg} /(\mathrm{kg} . \mathrm{d}) 8 \\
\text { weeks }\end{array}$ & $\begin{array}{l}\text { prednisolone } \\
\text { acetate } \\
1.5-2.0 \mathrm{mg} /(\mathrm{kg} . \mathrm{d}) 8 \\
\text { weeks }\end{array}$ & $(1)(2)(3)$ & $\begin{array}{l}\text { random number } \\
\text { table }\end{array}$ & $\begin{array}{l}\text { unclear } \\
\text { the exact } \\
\text { allocation }\end{array}$ & unclear & No one & 3 \\
\hline $\begin{array}{l}\text { Lin SX } \\
2015[43]\end{array}$ & \multicolumn{2}{|c|}{$161 / 424.2 \pm 0.6$} & $\begin{array}{l}\text { Yishen Jianpi Huoxue } \\
\text { Decoction+prednisone } \\
\text { 1.8-8.0mg/(kg.d) } 4 \text { weeks }\end{array}$ & $\begin{array}{l}\text { prednisone } \\
1.8-8.0 \mathrm{mg} /(\mathrm{kg} . \mathrm{d}) 4 \\
\text { weeks }\end{array}$ & $(1)(2)$ & $\begin{array}{l}\text { random number } \\
\text { table }\end{array}$ & $\begin{array}{l}\text { unclear } \\
\text { the exact } \\
\text { allocation }\end{array}$ & unclear & No one & 3 \\
\hline
\end{tabular}




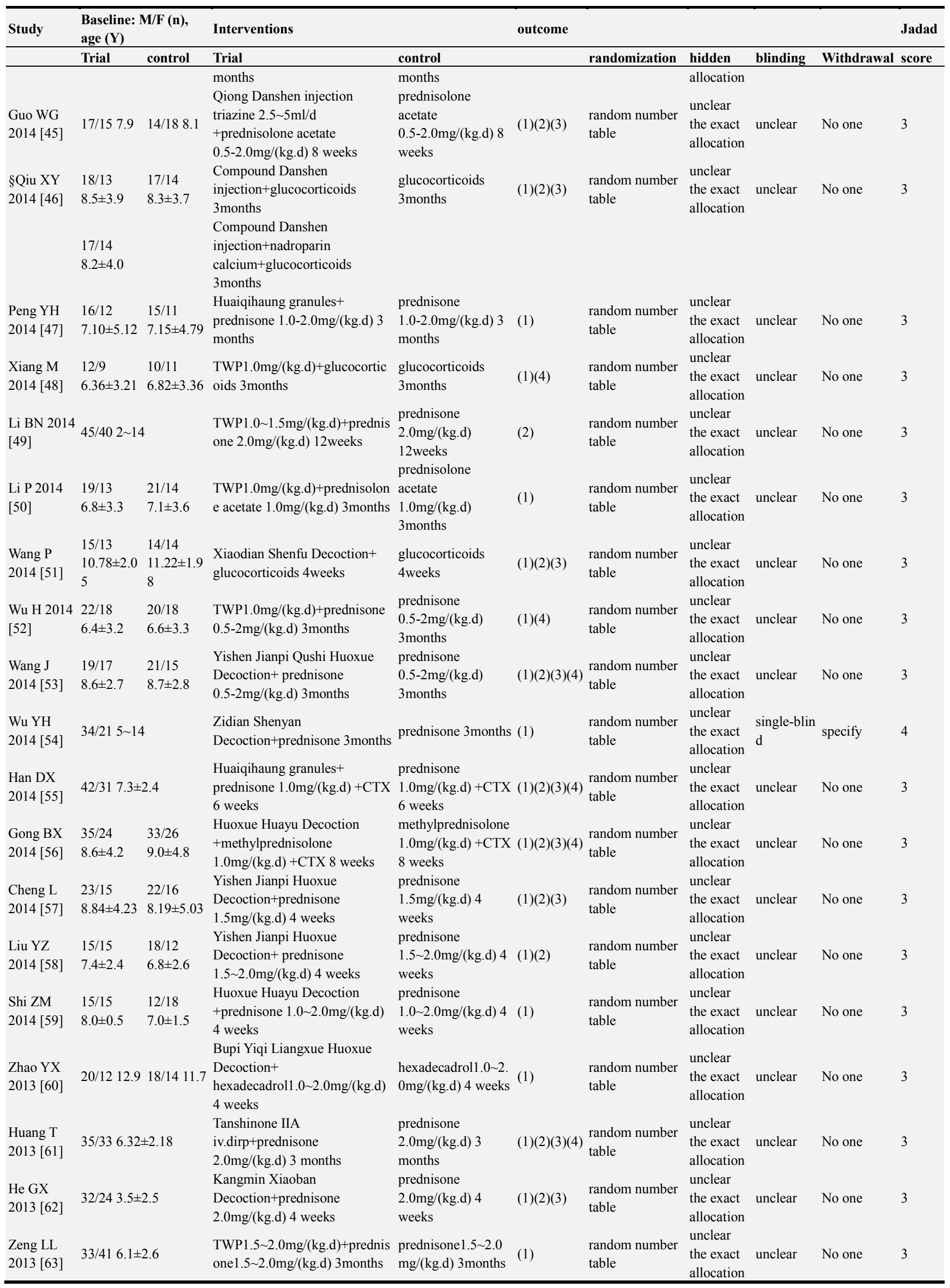




\begin{tabular}{|c|c|c|c|c|c|c|c|c|c|c|}
\hline Study & $\begin{array}{l}\text { Baseline: } \mathrm{N} \\
\text { age }(\mathrm{Y})\end{array}$ & $\mathbf{M} / \mathbf{F}(\mathbf{n})$ & Interventions & & outcome & & & & & Jadad \\
\hline & Trial & control & Trial & control & & randomization & hidden & blinding & Withdrawal & score \\
\hline $\begin{array}{l}\text { Yan HQ } \\
2013[64]\end{array}$ & $\begin{array}{l}24 / 21 \\
7.32 \pm 2.08\end{array}$ & $\begin{array}{l}25 / 20 \\
6.98 \pm 2.18\end{array}$ & $\begin{array}{l}\text { TWP } 1.0 \mathrm{mg} /(\mathrm{kg} . \mathrm{d})+\text { prednisone } \\
0.5 \sim 2.0 \mathrm{mg} /(\mathrm{kg} . \mathrm{d}) 3 \text { months }\end{array}$ & $\begin{array}{l}\text { prednisone } 0.5 \sim 2.0 \\
\mathrm{mg} /(\mathrm{kg} . \mathrm{d}) 3 \mathrm{months}\end{array}$ & $(1)(4)$ & $\begin{array}{l}\text { random number } \\
\text { table }\end{array}$ & $\begin{array}{l}\text { unclear } \\
\text { the exact } \\
\text { allocation }\end{array}$ & unclear & No one & 3 \\
\hline $\begin{array}{l}\text { Jiang JP } \\
2013[65]\end{array}$ & $\begin{array}{l}20 / 14 \\
7.23 \pm 3.53\end{array}$ & $\begin{array}{l}19 / 14 \\
7.09 \pm 3.84\end{array}$ & $\begin{array}{l}\text { Qingre Zhuyu } \\
\text { Decoction+prednisone } 0.5 \sim 2.0 \\
\text { mg/(kg.d) 3months }\end{array}$ & $\begin{array}{l}\text { prednisone } \\
0.5 \sim 2.0 \mathrm{mg} /(\mathrm{kg} . \mathrm{d}) \\
3 \text { months }\end{array}$ & (1) & $\begin{array}{l}\text { random number } \\
\text { table }\end{array}$ & $\begin{array}{l}\text { unclear } \\
\text { the exact } \\
\text { allocation }\end{array}$ & unclear & No one & 3 \\
\hline $\begin{array}{l}\text { Deng BZ } \\
2013[66]\end{array}$ & $10 / 613.8$ & 9/7 13.3 & $\begin{array}{l}\text { Xiaodian } \\
\text { Decoction+prednisone } 8 \text { weeks }\end{array}$ & prednisone 8 weeks & $(1)(2)(4)$ & $\begin{array}{l}\text { random number } \\
\text { table }\end{array}$ & $\begin{array}{l}\text { unclear } \\
\text { the exact } \\
\text { allocation }\end{array}$ & unclear & No one & 3 \\
\hline $\begin{array}{l}\text { Li MY } \\
2013[67]\end{array}$ & $14 / 126.2$ & $13 / 77.5$ & $\begin{array}{l}\text { Chinese herbs }+ \text { prednisone } \\
1.0 \sim 2.0 \mathrm{mg} /(\mathrm{kg} . \mathrm{d}) 8 \text { weeks }\end{array}$ & $\begin{array}{l}\text { prednisone } \\
1.0 \sim 2.0 \mathrm{mg} /(\mathrm{kg} . \mathrm{d}) 8 \\
\text { weeks }\end{array}$ & (1) & $\begin{array}{l}\text { random number } \\
\text { table }\end{array}$ & $\begin{array}{l}\text { unclear } \\
\text { the exact } \\
\text { allocation }\end{array}$ & unclear & No one & 3 \\
\hline $\begin{array}{l}\text { Jin JW } \\
2013[68]\end{array}$ & $\begin{array}{l}22 / 20 \\
9.0 \pm 1.8\end{array}$ & $\begin{array}{l}21 / 20 \\
9.1 \pm 1.8\end{array}$ & $\begin{array}{l}\text { Yuduqing } \\
\text { Decoction+glucocorticoids+CT } \\
\text { X 12weeks }\end{array}$ & $\begin{array}{l}\text { glucocorticoids }+\mathrm{CT} \\
\mathrm{X} 12 \text { weeks }\end{array}$ & (1) & $\begin{array}{l}\text { random number } \\
\text { table }\end{array}$ & $\begin{array}{l}\text { unclear } \\
\text { the exact } \\
\text { allocation }\end{array}$ & unclear & No one & 3 \\
\hline $\begin{array}{l}\text { Yuan PZ } \\
2013[69]\end{array}$ & $\begin{array}{l}19 / 15 \\
4 \sim 15\end{array}$ & $\begin{array}{l}20 / 15 \\
5 \sim 14\end{array}$ & $\begin{array}{l}\text { Zidian Shenkang } \\
\text { Decoction+glucocorticoids } \\
\text { 8weeks }\end{array}$ & $\begin{array}{l}\text { glucocorticoids } \\
\text { 8weeks }\end{array}$ & (1) & $\begin{array}{l}\text { random number } \\
\text { table }\end{array}$ & $\begin{array}{l}\text { unclear } \\
\text { the exact } \\
\text { allocation }\end{array}$ & unclear & No one & 3 \\
\hline $\begin{array}{l}\text { Wang YJ } \\
2012[70]\end{array}$ & $\begin{array}{l}30 / 20 \\
6.36 \pm 3.21\end{array}$ & $\begin{array}{l}26 / 28 \\
6.82 \pm 3.36\end{array}$ & $\begin{array}{l}\text { TWP } 1.0 \mathrm{mg} /(\mathrm{kg} . \mathrm{d})+\text { prednisone } \\
0.5 \sim 2.0 \mathrm{mg} /(\mathrm{kg} . \mathrm{d}) 3 \text { months }\end{array}$ & $\begin{array}{l}\text { prednisone } 0.5 \sim 2.0 \\
\mathrm{mg} /(\mathrm{kg} . \mathrm{d}) 3 \text { months }\end{array}$ & (1) & $\begin{array}{l}\text { random number } \\
\text { table }\end{array}$ & $\begin{array}{l}\text { unclear } \\
\text { the exact } \\
\text { allocation }\end{array}$ & unclear & No one & 3 \\
\hline $\begin{array}{l}\text { Ji RW } 2012 \\
{[71]}\end{array}$ & $21 / 1410.8$ & $20 / 159.6$ & $\begin{array}{l}\text { Yiqi Yangyang Huoxue } \\
\text { Decoction+ } \\
\text { prednisone } 1.0 \mathrm{mg} /(\mathrm{kg} . \mathrm{d}) 4 \\
\text { weeks }\end{array}$ & $\begin{array}{l}\text { prednisone } 1.0 \mathrm{mg} /(\mathrm{k} \\
\text { g.d) } 4 \text { weeks }\end{array}$ & (1) & $\begin{array}{l}\text { random number } \\
\text { table }\end{array}$ & $\begin{array}{l}\text { unclear } \\
\text { the exact } \\
\text { allocation }\end{array}$ & unclear & No one & 3 \\
\hline $\begin{array}{l}\text { Li YE } 2011 \\
{[72]}\end{array}$ & $18 / 1215$ & $19 / 1114$ & $\begin{array}{l}\text { Qingshen liangxue Decoction+ } \\
\text { prednisone } 1.0 \sim 2.0 \mathrm{mg} /(\mathrm{kg} . \mathrm{d}) 4 \\
\text { weeks }\end{array}$ & $\begin{array}{l}\text { prednisone } \\
1.0 \sim 2.0 \mathrm{mg} /(\mathrm{kg} . \mathrm{d}) 4 \\
\text { weeks }\end{array}$ & (1) & $\begin{array}{l}\text { random number } \\
\text { table }\end{array}$ & $\begin{array}{l}\text { unclear } \\
\text { the exact } \\
\text { allocation }\end{array}$ & unclear & No one & 3 \\
\hline $\begin{array}{l}\text { Chen CL } \\
2011[73]\end{array}$ & $\begin{array}{l}19 / 14 \\
8.1 \pm 3.7\end{array}$ & $\begin{array}{l}18 / 13 \\
8.7 \pm 2.9\end{array}$ & $\begin{array}{l}\text { Compound Danshen } \\
\text { injection+nadroparin } \\
\text { calcium+glucocorticoids } \\
3 \text { months }\end{array}$ & $\begin{array}{l}\text { glucocorticoids } \\
\text { 3months }\end{array}$ & (1) & $\begin{array}{l}\text { random number } \\
\text { table }\end{array}$ & $\begin{array}{l}\text { unclear } \\
\text { the exact } \\
\text { allocation }\end{array}$ & unclear & No one & 3 \\
\hline $\begin{array}{l}\text { Pang FM } \\
2011[74]\end{array}$ & $25 / 97.5$ & $23 / 78.0$ & $\begin{array}{l}\text { TWP } 1.0 \mathrm{mg} /(\mathrm{kg} . \mathrm{d})+\text { prednisone } 0 \\
.5 \sim 2.0 \mathrm{mg} /(\mathrm{kg} . \mathrm{d}) 3 \text { months }\end{array}$ & $\begin{array}{l}\text { prednisone } 0.5 \sim 2.0 \\
\mathrm{mg} /(\mathrm{kg} . \mathrm{d}) 3 \text { months }\end{array}$ & (1) & $\begin{array}{l}\text { random number } \\
\text { table }\end{array}$ & $\begin{array}{l}\text { unclear } \\
\text { the exact } \\
\text { allocation }\end{array}$ & unclear & No one & 3 \\
\hline $\begin{array}{l}\text { Pei HB } \\
2011[75]\end{array}$ & $\begin{array}{l}20 / 17 \\
7.0 \pm 1.0\end{array}$ & $\begin{array}{l}20 / 15 \\
7.2 \pm 1.2\end{array}$ & $\begin{array}{l}\text { Shenggyang Sanhuo } \\
\text { Decoction+prednisone } 1.0 \mathrm{mg} /(\mathrm{k} \\
\text { g.d) 4weeks }\end{array}$ & $\begin{array}{l}\text { prednisone } 1.0 \mathrm{mg} /(\mathrm{k} \\
\text { g.d) } 4 \text { weeks }\end{array}$ & $(1)(2)(4)$ & $\begin{array}{l}\text { random number } \\
\text { table }\end{array}$ & $\begin{array}{l}\text { unclear } \\
\text { the exact } \\
\text { allocation }\end{array}$ & unclear & No one & 3 \\
\hline $\begin{array}{l}\text { Wang CF } \\
2011[76]\end{array}$ & $\begin{array}{l}128 / 84 \\
10.1 \pm 3.27\end{array}$ & $\begin{array}{l}114 / 98 \\
9.5 \pm 3.87\end{array}$ & $\begin{array}{l}\text { Yishen } \\
\text { granules+glucocorticoids+CTX } \\
6 \text { months }\end{array}$ & $\begin{array}{l}\text { glucocorticoids }+\mathrm{CT} \\
\text { X } 6 \text { months }\end{array}$ & (1) & $\begin{array}{l}\text { random number } \\
\text { table }\end{array}$ & $\begin{array}{l}\text { unclear } \\
\text { the exact } \\
\text { allocation }\end{array}$ & unclear & No one & 3 \\
\hline $\begin{array}{l}\text { Guo YH } \\
2011[77]\end{array}$ & $27 / 334 \sim 14$ & & $\begin{array}{l}\text { Zidian Shenyan } \\
\text { Decoction+prednisone } \\
0.5 \sim 2.0 \mathrm{mg} /(\mathrm{kg} . \mathrm{d}) 3 \text { months }\end{array}$ & $\begin{array}{l}\text { prednisone } \\
0.5 \sim 2.0 \mathrm{mg} /(\mathrm{kg} . \mathrm{d}) \\
3 \text { months }\end{array}$ & (1) & $\begin{array}{l}\text { random number } \\
\text { table }\end{array}$ & $\begin{array}{l}\text { unclear } \\
\text { the exact } \\
\text { allocation }\end{array}$ & unclear & No one & 3 \\
\hline $\begin{array}{l}\text { Li JC } 2011 \\
{[78]}\end{array}$ & $\begin{array}{l}18 / 12 \\
5 \sim 14\end{array}$ & $\begin{array}{l}16 / 14 \\
5 \sim 14\end{array}$ & $\begin{array}{l}\text { Chinese herbs + glucocorticoids } \\
1 \text { month }\end{array}$ & $\begin{array}{l}\text { glucocorticoids } \\
\text { 1month }\end{array}$ & (1) & $\begin{array}{l}\text { random number } \\
\text { table }\end{array}$ & $\begin{array}{l}\text { unclear } \\
\text { the exact } \\
\text { allocation }\end{array}$ & unclear & No one & 3 \\
\hline $\begin{array}{l}\text { Zhao ZY } \\
2011 \text { [79] }\end{array}$ & $26 / 148.2$ & $12 / 88.1$ & $\begin{array}{l}\text { Yishen } \\
\text { Decoction+glucocorticoids+CT } \\
\text { X 12weeks }\end{array}$ & $\begin{array}{l}\text { glucocorticoids }+\mathrm{CT} \\
\mathrm{X} 12 \text { weeks }\end{array}$ & $(1)(2)(3)$ & $\begin{array}{l}\text { random number } \\
\text { table }\end{array}$ & $\begin{array}{l}\text { unclear } \\
\text { the exact } \\
\text { allocation }\end{array}$ & unclear & No one & 3 \\
\hline $\begin{array}{l}\text { Peng HP } \\
2011[80]\end{array}$ & $17 / 332 \sim 15$ & & $\begin{array}{l}\text { Chinese herbs } \\
\text { +glucocorticoids+CTX 4weeks }\end{array}$ & $\begin{array}{l}\text { glucocorticoids }+\mathrm{CT} \\
\mathrm{X} 4 \text { weeks }\end{array}$ & (1) & $\begin{array}{l}\text { random number } \\
\text { table }\end{array}$ & $\begin{array}{l}\text { unclear } \\
\text { the exact } \\
\text { allocation }\end{array}$ & unclear & No one & 3 \\
\hline $\begin{array}{l}\text { Zhao YD } \\
2011 \text { [81] }\end{array}$ & $\begin{array}{l}16 / 14 \\
3 \sim 16\end{array}$ & $\begin{array}{l}19 / 11 \\
3 \sim 16\end{array}$ & $\begin{array}{l}\text { Chinese herbs }+ \text { prednisone } \\
1.0 \mathrm{mg} /(\mathrm{kg} . \mathrm{d}) 2 \text { months }\end{array}$ & $\begin{array}{l}\text { prednisone } \\
1.0 \mathrm{mg} /(\mathrm{kg} . \mathrm{d}) 2 \\
\text { months }\end{array}$ & $(1)(4)$ & $\begin{array}{l}\text { random number } \\
\text { table }\end{array}$ & $\begin{array}{l}\text { unclear } \\
\text { the exact } \\
\text { allocation }\end{array}$ & unclear & No one & 3 \\
\hline $\begin{array}{l}\text { Hao JJ } \\
2011[82]\end{array}$ & $\begin{array}{l}60 / 24 \\
9.7 \pm 3.9\end{array}$ & $\begin{array}{l}54 / 24 \\
8.9 \pm 4.8\end{array}$ & $\begin{array}{l}\text { TWP } 1.5 \mathrm{mg} /(\mathrm{kg} . \mathrm{d})+\text { prednisone } \\
0.5 \sim 2.0 \mathrm{mg} /(\mathrm{kg} . \mathrm{d}) 4 \text { months }\end{array}$ & $\begin{array}{l}\text { prednisone } 0.5 \sim 2.0 \\
\mathrm{mg} /(\mathrm{kg} . \mathrm{d}) 4 \text { months }\end{array}$ & $(1)(2)(3)$ & $\begin{array}{l}\text { random number } \\
\text { table }\end{array}$ & $\begin{array}{l}\text { unclear } \\
\text { the exact } \\
\text { allocation }\end{array}$ & unclear & No one & 3 \\
\hline $\begin{array}{l}\mathrm{Lu} J 2010 \\
{[83]}\end{array}$ & $25 / 178.5$ & $23 / 179.1$ & $\begin{array}{l}\text { Liangxue Huoxue Huayu } \\
\text { Decoction+ glucocorticoids } \\
\text { 4weeks }\end{array}$ & $\begin{array}{l}\text { glucocorticoids } \\
\text { 4weeks }\end{array}$ & (1) & $\begin{array}{l}\text { random number } \\
\text { table }\end{array}$ & $\begin{array}{l}\text { unclear } \\
\text { the exact } \\
\text { allocation }\end{array}$ & unclear & No one & 3 \\
\hline $\begin{array}{l}\text { Chen ZH } \\
2010[84]\end{array}$ & $\begin{array}{l}22 / 16 \\
8.3 \pm 2.6\end{array}$ & $\begin{array}{l}18 / 14 \\
8.2 \pm 2.8\end{array}$ & $\begin{array}{l}\text { Chinese herbs }+ \text { prednisone } \\
1.0 \sim 2.0 \mathrm{mg} /(\mathrm{kg} . \mathrm{d}) 4 \text { weeks }\end{array}$ & $\begin{array}{l}\text { prednisone } \\
1.0 \sim 2.0 \mathrm{mg} /(\mathrm{kg} . \mathrm{d}) 4 \\
\text { weeks }\end{array}$ & $(1)(2)(3)(4)$ & $\begin{array}{l}\text { random number } \\
\text { table }\end{array}$ & $\begin{array}{l}\text { unclear } \\
\text { the exact } \\
\text { allocation }\end{array}$ & unclear & No one & 3 \\
\hline
\end{tabular}




\begin{tabular}{|c|c|c|c|c|c|c|c|c|c|c|}
\hline Study & $\begin{array}{l}\text { Baseline: } 1 \\
\text { age }(Y)\end{array}$ & $\mathbf{M} / \mathbf{F}(\mathbf{n})$ & Interventions & & outcome & & & & & Jadad \\
\hline & Trial & control & Trial & control & & randomization & hidden & blinding & Withdrawal & score \\
\hline $\begin{array}{l}\text { Yu FM } \\
2010[85]\end{array}$ & $12 / 1614$ & $17 / 1114$ & $\begin{array}{l}\text { Zidian Decoction+prednisone } \\
1.0 \mathrm{mg} /(\mathrm{kg} . \mathrm{d}) 4 \text { weeks }\end{array}$ & $\begin{array}{l}\text { prednisone } \\
1.0 \mathrm{mg} /(\mathrm{kg} . \mathrm{d}) 4 \\
\text { weeks }\end{array}$ & (1) & $\begin{array}{l}\text { random number } \\
\text { table }\end{array}$ & $\begin{array}{l}\text { unclear } \\
\text { the exact } \\
\text { allocation }\end{array}$ & unclear & No one & 3 \\
\hline $\begin{array}{l}\text { Zhang TD } \\
2010[86]\end{array}$ & $18 / 1215$ & $19 / 1114$ & $\begin{array}{l}\text { Xijiao Dihaung Decoction } \\
\text { +prednisone } 1.0 \sim 2.0 \mathrm{mg} /(\mathrm{kg} . \mathrm{d}) \\
4 \text { weeks }\end{array}$ & $\begin{array}{l}\text { prednisone } \\
1.0 \sim 2.0 \mathrm{mg} /(\mathrm{kg} . \mathrm{d}) 4 \\
\text { weeks }\end{array}$ & (1) & $\begin{array}{l}\text { random number } \\
\text { table }\end{array}$ & $\begin{array}{l}\text { unclear } \\
\text { the exact } \\
\text { allocation }\end{array}$ & unclear & No one & 3 \\
\hline $\begin{array}{l}\text { Guo QY } \\
2009 \text { [87] }\end{array}$ & $\begin{array}{l}30 / 12 \\
9.7 \pm 3.9\end{array}$ & $\begin{array}{l}27 / 12 \\
8.9 \pm 4.8\end{array}$ & $\begin{array}{l}\text { TWP+Chinese herbs } \\
\text { + prednisone } 2.0 \mathrm{mg} /(\mathrm{kg} . \mathrm{d}) 4 \\
\text { months }\end{array}$ & $\begin{array}{l}\text { prednisone } \\
2.0 \mathrm{mg} /(\mathrm{kg} . \mathrm{d}) 4 \\
\text { months }\end{array}$ & $(1)(2)(3)$ & $\begin{array}{l}\text { random number } \\
\text { table }\end{array}$ & $\begin{array}{l}\text { unclear } \\
\text { the exact } \\
\text { allocation }\end{array}$ & unclear & No one & 3 \\
\hline $\begin{array}{l}\text { Huang QY } \\
2009[88]\end{array}$ & $\begin{array}{l}18 / 13 \\
7.9 \pm 2.4\end{array}$ & $\begin{array}{l}13 / 17 \\
8.5 \pm 2.3\end{array}$ & $\begin{array}{l}\text { TWP+nadroparin } \\
\text { calcium+glucocorticoids } 2 \\
\text { weeks }\end{array}$ & $\begin{array}{l}\text { glucocorticoids } 2 \\
\text { weeks }\end{array}$ & (1) & $\begin{array}{l}\text { random number } \\
\text { table }\end{array}$ & $\begin{array}{l}\text { unclear } \\
\text { the exact } \\
\text { allocation }\end{array}$ & unclear & No one & 3 \\
\hline $\begin{array}{l}\text { Chen CB } \\
2009 \text { [89] }\end{array}$ & $10 / 78.2$ & $10 / 98.0$ & $\begin{array}{l}\text { TWP+glucocorticoids }+ \text { CTX } 3 \\
\text { momths }\end{array}$ & $\begin{array}{l}\text { glucocorticoids }+\mathrm{CT} \\
\text { X } 3 \text { momths }\end{array}$ & (1)(4) & $\begin{array}{l}\text { random number } \\
\text { table }\end{array}$ & $\begin{array}{l}\text { unclear } \\
\text { the exact } \\
\text { allocation }\end{array}$ & unclear & No one & 3 \\
\hline $\begin{array}{l}\text { Ma XC } \\
2009[90]\end{array}$ & $\begin{array}{l}17 / 13 \\
2 \sim 15\end{array}$ & $\begin{array}{l}10 / 10 \\
2 \sim 15\end{array}$ & $\begin{array}{l}\text { Shenyankang } \\
\text { granule+glucocorticoids } 4 \\
\text { weeks }\end{array}$ & $\begin{array}{l}\text { glucocorticoids } 4 \\
\text { weeks }\end{array}$ & (1) & $\begin{array}{l}\text { random number } \\
\text { table }\end{array}$ & $\begin{array}{l}\text { unclear } \\
\text { the exact } \\
\text { allocation }\end{array}$ & unclear & No one & 3 \\
\hline $\begin{array}{l}\mathrm{Li} \mathrm{G} 2009 \\
{[91]}\end{array}$ & $17 / 710$ & & $\begin{array}{l}\text { Chinese herbs }+ \text { glucocorticoids } \\
4 \text { weeks }\end{array}$ & $\begin{array}{l}\text { glucocorticoids } 4 \\
\text { weeks }\end{array}$ & (1) & $\begin{array}{l}\text { random number } \\
\text { table }\end{array}$ & $\begin{array}{l}\text { unclear } \\
\text { the exact } \\
\text { allocation }\end{array}$ & unclear & No one & 3 \\
\hline $\begin{array}{l}\text { Huang WY } \\
2009 \text { [92] }\end{array}$ & $\begin{array}{l}21 / 14 \\
5 \sim 15\end{array}$ & $\begin{array}{l}18 / 12 \\
4 \sim 14\end{array}$ & $\begin{array}{l}\text { Chinese herbs }+ \text { glucocorticoids } \\
4 \text { weeks }\end{array}$ & $\begin{array}{l}\text { glucocorticoids } 4 \\
\text { weeks }\end{array}$ & (1) & $\begin{array}{l}\text { random number } \\
\text { table }\end{array}$ & $\begin{array}{l}\text { unclear } \\
\text { the exact } \\
\text { allocation }\end{array}$ & unclear & No one & 3 \\
\hline $\begin{array}{l}\text { Wu ZC } \\
2009 \text { [93] }\end{array}$ & $50 / 429.2$ & & $\begin{array}{l}\text { Chinese herbs }+ \text { prednisone } \\
2.0 \mathrm{mg} /(\mathrm{kg} . \mathrm{d}) 10 \text { days }\end{array}$ & $\begin{array}{l}\text { prednisone } \\
2.0 \mathrm{mg} /(\mathrm{kg} . \mathrm{d}) 10 \\
\text { days }\end{array}$ & (1) & $\begin{array}{l}\text { random number } \\
\text { table }\end{array}$ & $\begin{array}{l}\text { unclear } \\
\text { the exact } \\
\text { allocation }\end{array}$ & unclear & No one & 3 \\
\hline $\begin{array}{l}\text { Wang J } \\
2009 \text { [94] }\end{array}$ & $\begin{array}{l}17 / 11 \\
7.8 \pm 2.6\end{array}$ & $\begin{array}{l}18 / 12 \\
8.2 \pm 2.8\end{array}$ & $\begin{array}{l}\text { Yishen Jianpi Huoxue } \\
\text { Decoction+ prednisone } \\
1.5 \sim 2.0 \mathrm{mg} /(\mathrm{kg} . \mathrm{d}) 4 \text { weeks }\end{array}$ & $\begin{array}{l}\text { prednisone } \\
1.5 \sim 2.0 \mathrm{mg} /(\mathrm{kg} . \mathrm{d}) 4 \\
\text { weeks }\end{array}$ & $(1)(2)(3)$ & $\begin{array}{l}\text { random number } \\
\text { table }\end{array}$ & $\begin{array}{l}\text { unclear } \\
\text { the exact } \\
\text { allocation }\end{array}$ & unclear & No one & 3 \\
\hline $\begin{array}{l}\text { Wang YL } \\
2007[96]\end{array}$ & $\begin{array}{l}33 / 23 \\
7.20 \pm 3.92\end{array}$ & $\begin{array}{l}29 / 21 \\
7.90 \pm 3.58\end{array}$ & $\begin{array}{l}\text { TWP } 1.0 \mathrm{mg} /(\mathrm{kg} . \mathrm{d})+\text { prednisone } \\
0.5 \sim 2.0 \mathrm{mg} /(\mathrm{kg} . \mathrm{d}) 12 \text { weeks }\end{array}$ & $\begin{array}{l}\text { prednisone } 0.5 \sim 2.0 \\
\mathrm{mg} /(\mathrm{kg} . \mathrm{d}) 12 \text { weeks }\end{array}$ & (1) & $\begin{array}{l}\text { random number } \\
\text { table }\end{array}$ & $\begin{array}{l}\text { unclear } \\
\text { the exact } \\
\text { allocation }\end{array}$ & unclear & No one & 3 \\
\hline $\begin{array}{l}\text { Fan WW } \\
2007[97]\end{array}$ & $15 / 88.2$ & $13 / 89.1$ & $\begin{array}{l}\text { Qingre Huayu Shufeng } \\
\text { Decoction+prednisone } \\
0.8 \sim 1.0 \mathrm{mg} /(\mathrm{kg} . \mathrm{d}) 3 \text { months }\end{array}$ & $\begin{array}{l}\text { prednisone } \\
0.8 \sim 1.0 \mathrm{mg} /(\mathrm{kg} . \mathrm{d}) 3 \\
\text { months }\end{array}$ & $(1)(2)(3)$ & $\begin{array}{l}\text { random number } \\
\text { table }\end{array}$ & $\begin{array}{l}\text { unclear } \\
\text { the exact } \\
\text { allocation }\end{array}$ & unclear & No one & 3 \\
\hline $\begin{array}{l}\text { Zhang JP } \\
2006 \text { [98] }\end{array}$ & $15 / 117.4$ & $14 / 137.6$ & $\begin{array}{l}\text { TWP } 1.0 \mathrm{mg} /(\mathrm{kg} . \mathrm{d})+\text { prednisone } \\
1.5 \sim 2.0 \mathrm{mg} /(\mathrm{kg} . \mathrm{d}) 12 \text { weeks }\end{array}$ & $\begin{array}{l}\text { prednisone } 1.5 \sim 2.0 \\
\mathrm{mg} /(\mathrm{kg} . \mathrm{d}) 12 \text { weeks }\end{array}$ & (1) & $\begin{array}{l}\text { random number } \\
\text { table }\end{array}$ & $\begin{array}{l}\text { unclear } \\
\text { the exact } \\
\text { allocation }\end{array}$ & unclear & No one & 3 \\
\hline $\begin{array}{l}\text { Chang WJ } \\
2006 \text { [99] }\end{array}$ & $\begin{array}{l}46 / 14 \\
4 \sim 15\end{array}$ & $22 / 85 \sim 16$ & $\begin{array}{l}\text { Yiqi Tonglin } \\
\text { granules+glucocorticoids } 3 \\
\text { months }\end{array}$ & $\begin{array}{l}\text { glucocorticoids } 3 \\
\text { months }\end{array}$ & $(1)(2)(3)$ & $\begin{array}{l}\text { random number } \\
\text { table }\end{array}$ & $\begin{array}{l}\text { unclear } \\
\text { the exact } \\
\text { allocation }\end{array}$ & unclear & No one & 3 \\
\hline $\begin{array}{l}\text { Song MS } \\
2005[100]\end{array}$ & $\begin{array}{l}18 / 12 \\
9 \sim 13\end{array}$ & $\begin{array}{l}19 / 11 \\
8 \sim 14\end{array}$ & $\begin{array}{l}\text { Xiere Xiaoban } \\
\text { Decoction+glucocorticoids }\end{array}$ & glucocorticoids & $(1)(2)$ & $\begin{array}{l}\text { random number } \\
\text { table }\end{array}$ & $\begin{array}{l}\text { unclear } \\
\text { the exact } \\
\text { allocation }\end{array}$ & unclear & No one & 3 \\
\hline $\begin{array}{l}\text { Mao YY } \\
2004[101]\end{array}$ & $7 / 108.3$ & $6 / 98.6$ & $\begin{array}{l}\text { Zidian Shenyan } \\
\text { Decoction+prednisone } \\
1.5 \sim 2.0 \mathrm{mg} /(\mathrm{kg} . \mathrm{d})+\mathrm{CTX} 4 \\
\text { weeks }\end{array}$ & $\begin{array}{l}\text { prednisone } \\
1.5 \sim 2.0 \mathrm{mg} /(\mathrm{kg} . \mathrm{d})+ \\
\text { CTX } 4 \text { weeks }\end{array}$ & $(1)(2)(3)$ & $\begin{array}{l}\text { random number } \\
\text { table }\end{array}$ & $\begin{array}{l}\text { unclear } \\
\text { the exact } \\
\text { allocation }\end{array}$ & unclear & No one & 3 \\
\hline
\end{tabular}

(1): total effective rate (2): 24-hour urine protein ration (3): Urine erythrocyte count (4): adverse effects rate.

$\S$ three arms trial.

\subsection{Meta-Analysis}

\subsubsection{Effect of Combine Therapy on Total Effective Rates}

89 studies described efficiency, we combined and tested the results by the escalc functions of the $\mathrm{R}$ metafor. As presented in figure 2 , the forestplot showed the heterogeneity of each study $\left(\mathrm{P}<0.01, \mathrm{I}^{2}=38.98 \%\right)$, and the random effect model was used for analysis. Children treated with Chinese plus Western medicine demonstrated a significant increase in total effective rates [Figure $2 \mathrm{RR}=1.19 ; 95 \% \mathrm{CI}$ (1.16-1.21), $\mathrm{P}<0.001]$ when compared with Western medicine alone. Taking total effective rate as an evaluation index, children seemed to benefit more from combined therapy. Then the 91 studies were analyzed by funnel plot with regtest function, as presented in Figure 3, $\mathrm{P}<0.01$, the plot is not completely symmetrical, indicating publication bias. 


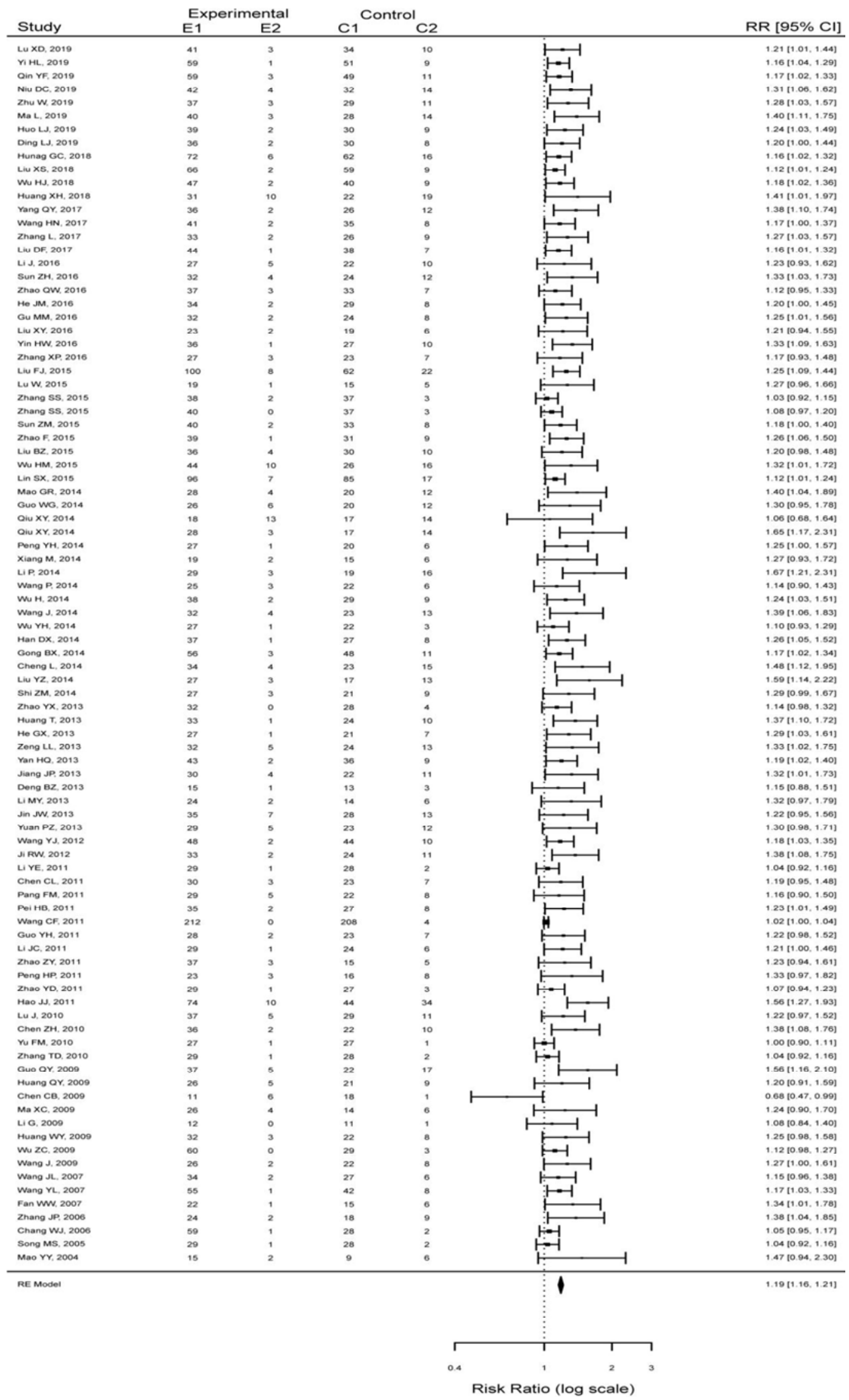

Figure 2. Forestplot for effective rate. 
Random-Effects Model

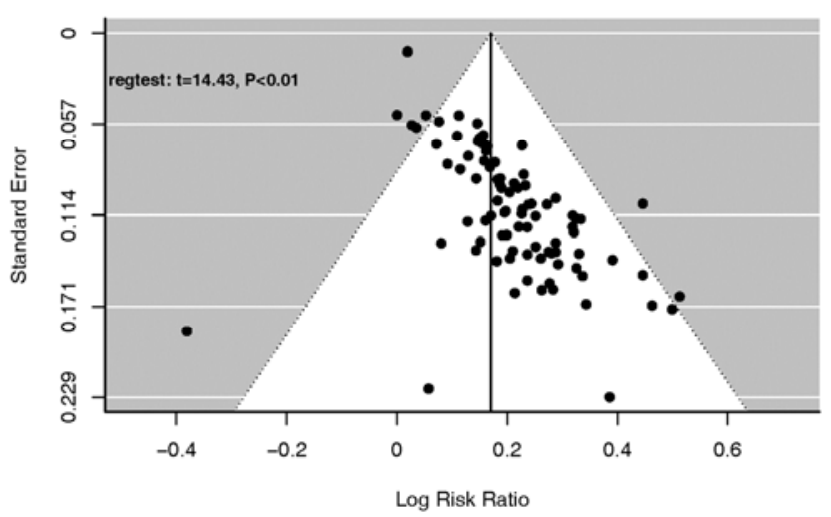

\subsubsection{Effect of Combined Therapy on 24-hour Urine Protein Ration Level}

37 studies described 24-hour urine protein ration level, we combined and tested the results by the metacont function of the $\mathrm{R}$ metafor. As presented in figure 4, the forestplot showed the heterogeneity of each study $\left(\mathrm{I}^{2}=98 \%\right)$, and the random effect model was used for analysis. Our study also found that Chinese plus Western medicine had an advantage on decreasing 24-hour urine protein [Figure $4 \mathrm{WMD}=-0.47$; $95 \% \mathrm{CI}(-0.53,-0.41) ; \mathrm{p}<0.001]$ when compared with Western medicine alone. We conducted funnel plot analysis of the 37 studies, as presented in Figure 5, $\mathrm{P}<0.001$, the plot is not completely symmetrical, indicating publication bias.

Figure 3. Funnelplot for effective rate.

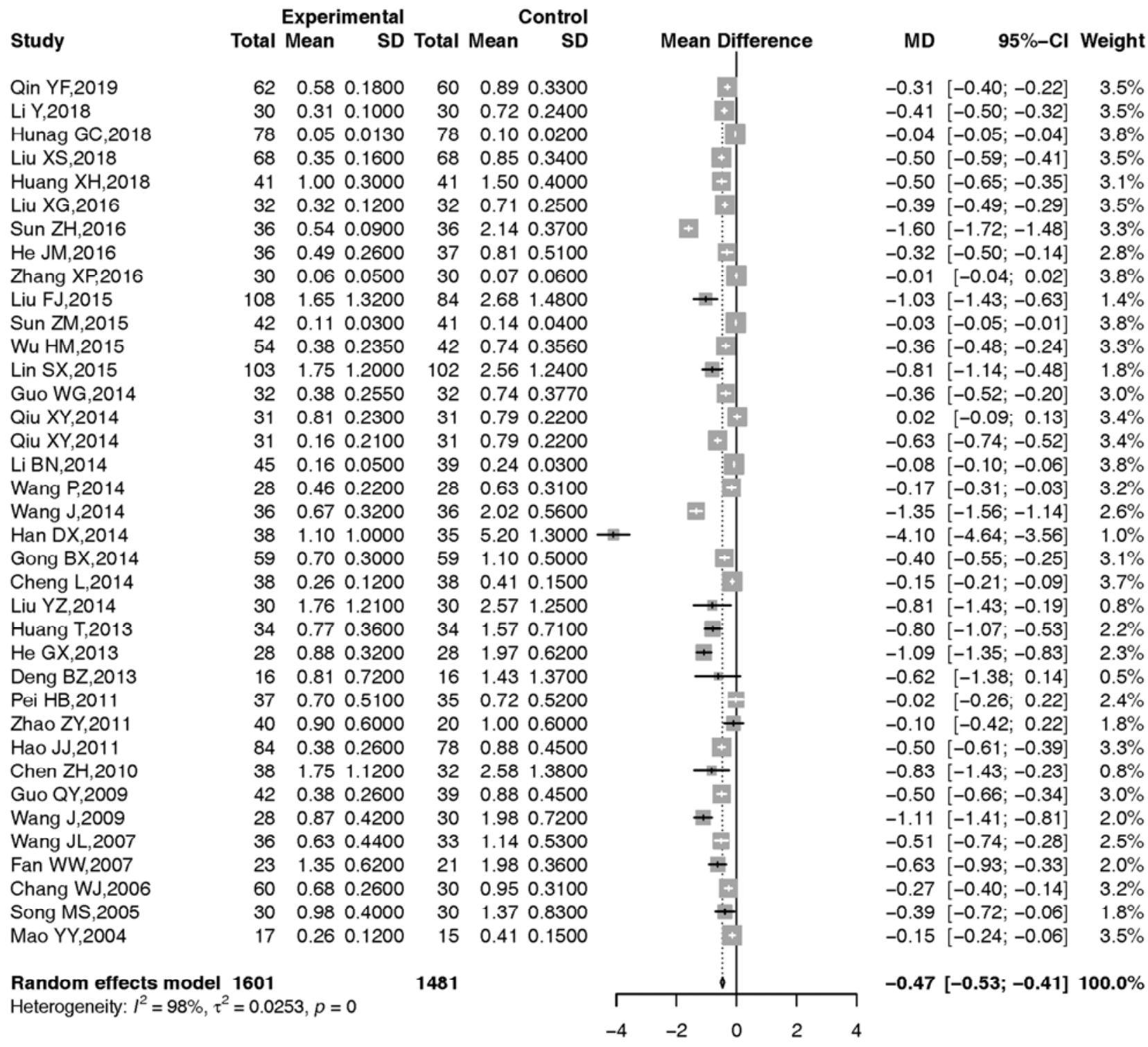

Figure 4. Forestplot for 24-hour urine protein. 


\section{Random-Effects Model}

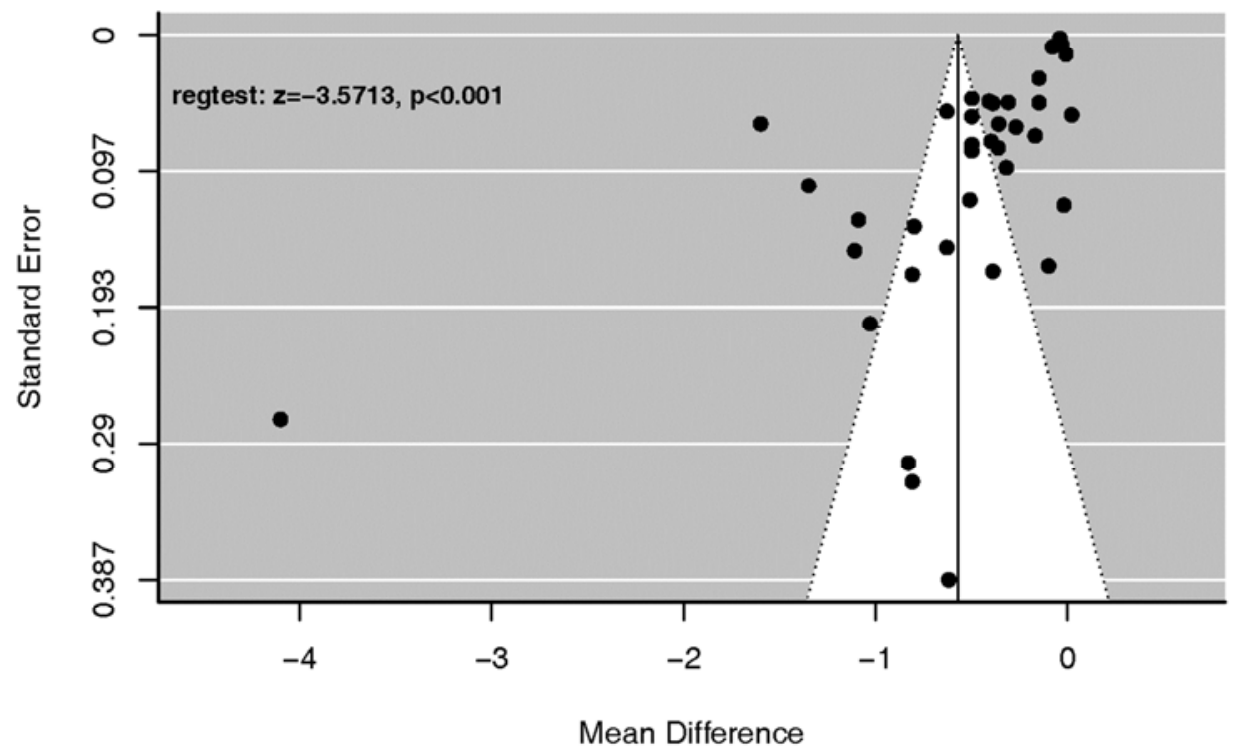

Figure 5. Funnelplot for 24-hour urine protein.

\subsubsection{Effect of Combined Therapy on Urine Erythrocyte Count Level}

We combined and tested the Urine erythrocyte count of 28 studies by the metacont function of the R metafor. Our study found combined therapy could significantly reduce the urine erythrocyte count [Figure $6 \mathrm{WMD}=-8.88 ; 95 \% \mathrm{CI}(-9.80,-7.96)$; $\mathrm{p}<0.001$, random effects model] compared with the western medicine alone. Funnel plot shows that the graph is not completely symmetrical $(\mathrm{P}=0.0119)$, Figure 7 , with publication bias.

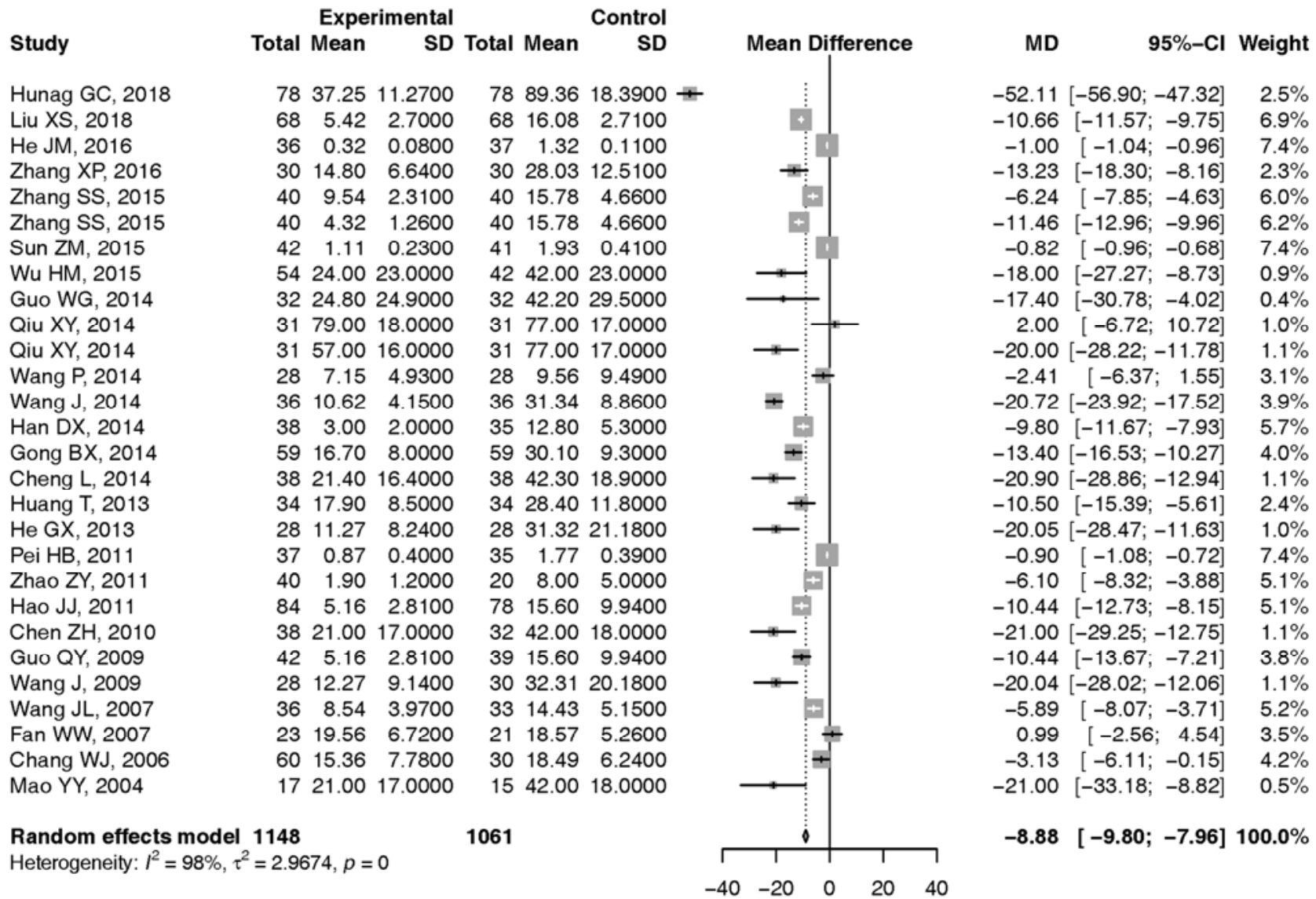

Figure 6. Forestplot for urine erythrocyte count. 


\section{Random-Effects Model}

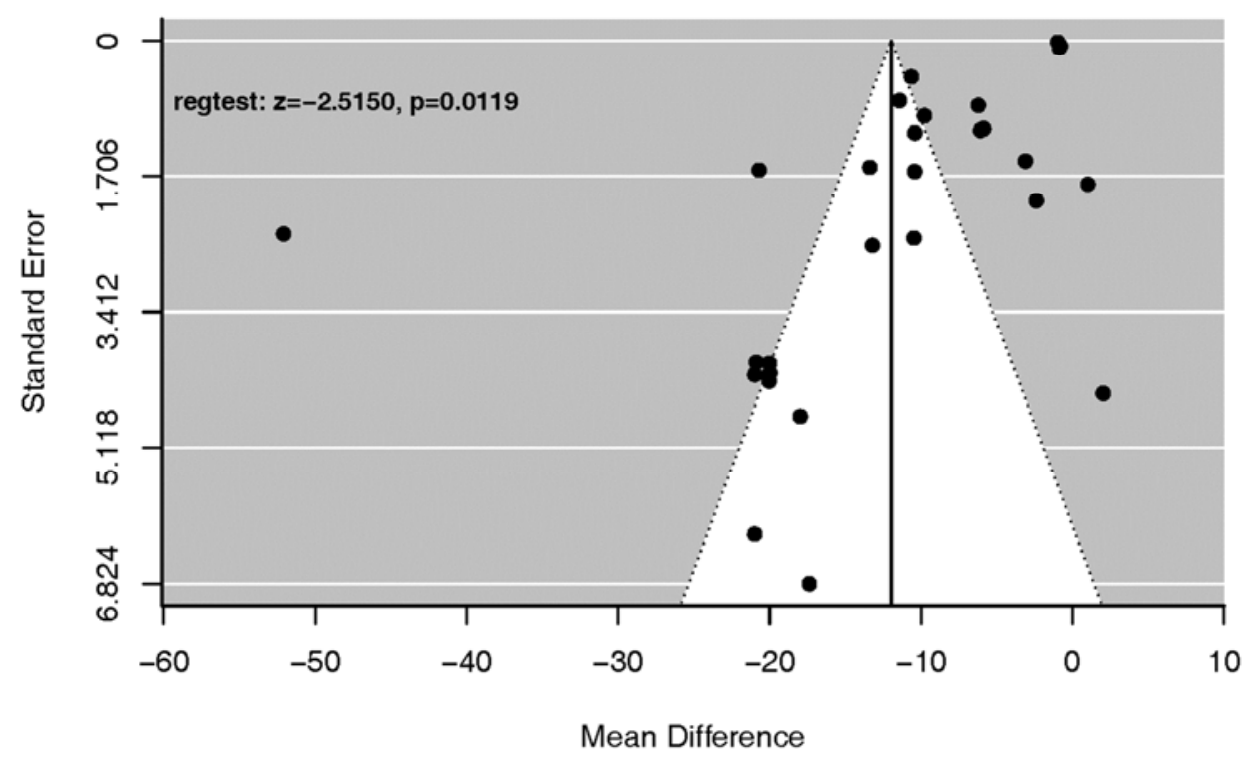

Figure 7. Funnelplot for urine erythrocyte count.

\subsubsection{Adverse Effects of the Combine Therapy}

30 studies reported adverse effect in the course of treatment. Heterogeneity exists in each study $(\mathrm{P}<0.01)$, the random effect model was adopted. It is proved that Chinese plus Western medicine could decrease adverse effects more effectively [Figure 8 $\mathrm{RR}=0.64 ; 95 \% \mathrm{CI}(0.46,0.87) ; \mathrm{p}<0.01]$ than Western medicine alone. The funnel plot also shows the graph is basically symmetrical $(\mathrm{P}=0.71>0.05)$, figure 9 , with no publication bias.

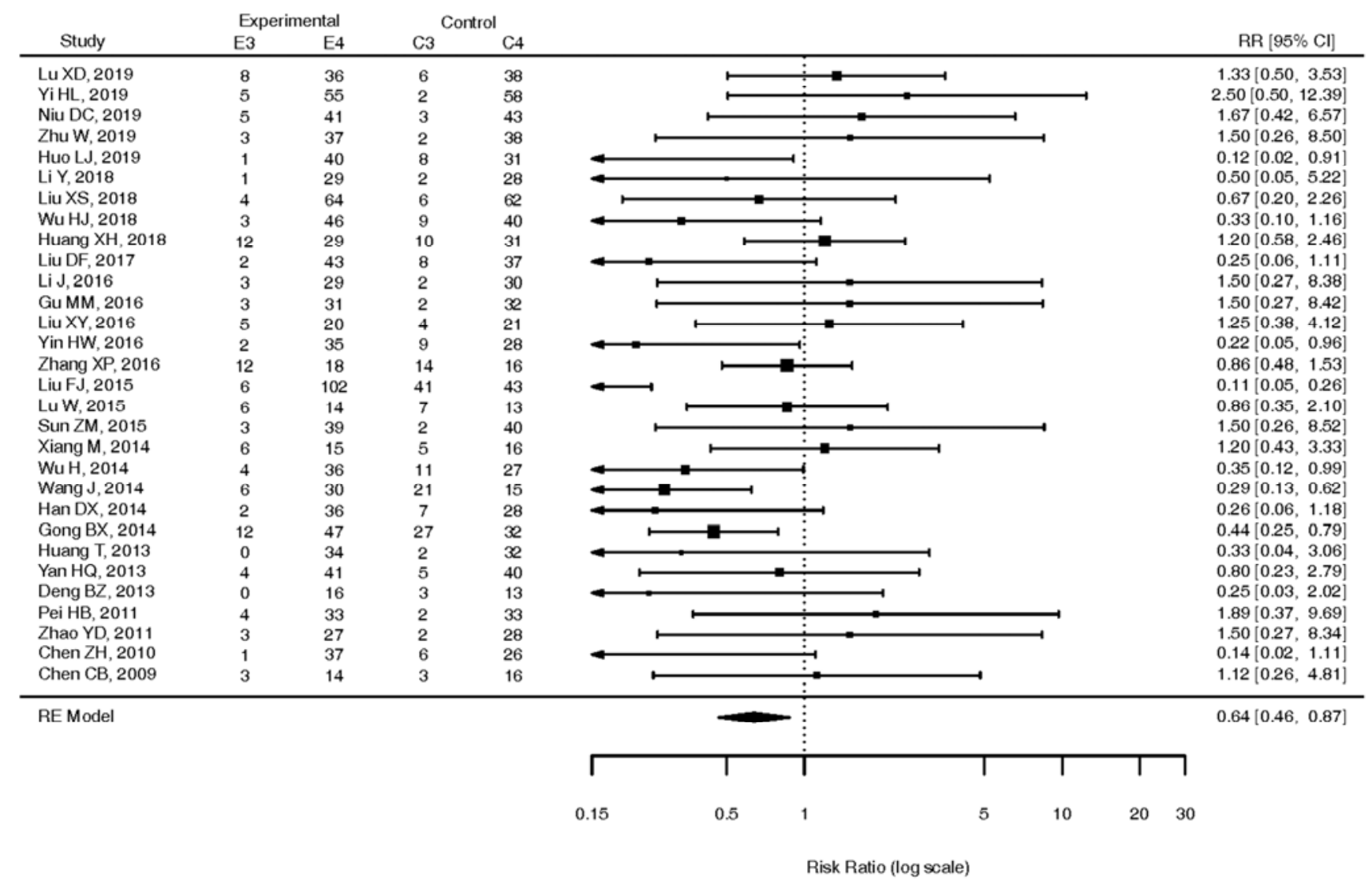

Figure 8. Forestplot for adverse rate. 


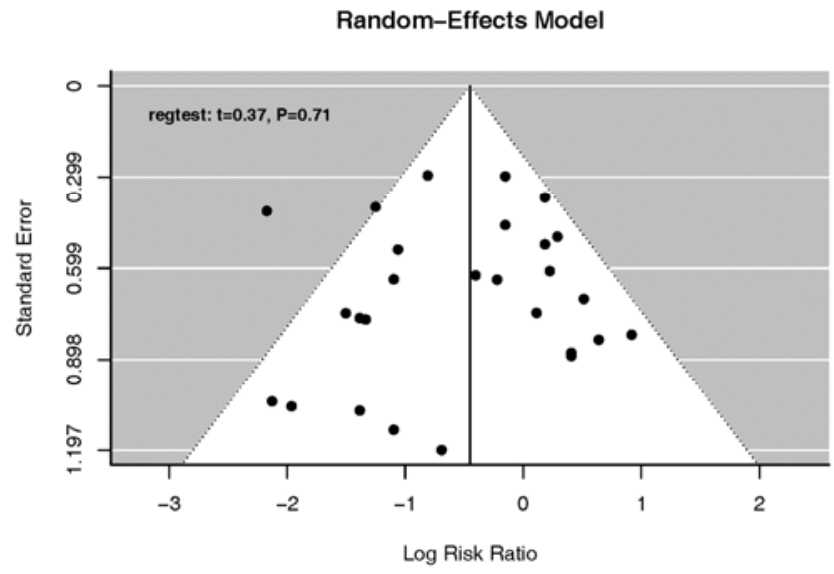

Figure 9. Funnelplot for adverse rate.

\subsubsection{Heterogeneity Analysis}

The main indicators, 24-hour urine protein ration, urine erythrocyte count, and adverse effect all showed high heterogeneity. We used the ratio of the number of men in the experimental group to the control group and the year of publication to fit the mixed effect model. 24-hour urine protein ration indicator shows two concomitant variables did not contribute to heterogeneity; urine erythrocyte count indicator shows two concomitant variables can explain the total heterogeneity of $5.78 \%$, but two concomitant variable are meaningless in the model; adverse effect indicator shows two concomitant variables can explain the total heterogeneity of $4.06 \%$, but two concomitant variables are also meaningless in the model. We tried unsuccessfully to find the source of heterogeneity. But the age and disease course of each experimental patient were basically the same between the two groups, the cause of heterogeneity is not clear, and it is preliminarily inferred that heterogeneity comes from the variation in the study.

\section{Conclusion}

The analysis in this study found that the integrated Chinese and Western medicine treatment for children who have HSPN was more superior in the total effective rate. Our studies also found the combined therapy could reduce the 24-hour urine protein ration level, Urine erythrocyte count and adverse rate, children could seemed to benefit more from combined treatment than the Western medicine alone. Integrated Chinese plus Western medicine treatment was effective for Childhood purpuric nephritis, which may be a superior alternative for HSPN. Nevertheless, the funnel plots of the total effective rate, 24-hour urine protein ration level and Urine erythrocyte count showed that the graphs are not completely symmetrical, with publication bias. So the long-term and high-quality RCTs are required to make the results more convincing.

At last, the main indicators, 24-hour urine protein ration, urine erythrocyte count and adverse effect all showed high heterogeneity. We performed a heterogeneity analysis from the ratio of the number of men and the year of publication using the mixed effect model, but the cause of heterogeneity is not clear, it is preliminarily inferred that heterogeneity comes from the variation in the study.

\section{Discussion}

The cause of HSPN is still unknown. At present, the etiology of HSPN is mainly attributive to infection factors and genetic factors. For example, viruses, bacteria, especially streptococcus and helicobacter pylori are more inclined to cause HSPN. The rubella, hepatitis virus, mycoplasma pneumoniae and parasitic infection may cause HSPN morbidity. In addition, HLA gene, IgA are also involved in the pathogenesis of HSPN [102]. In the theory of Chinese medicine, HSPN belongs to the category of "purpura, blood disorder". The clinical physicians are intended to type the HSPN into syndrome of heat in blood, the syndrome of qi deficiency, the syndrome of yin deficiency, the syndrome of dampness-heat blocking collaterals, the syndrome of deficiency of both qi and yin, the syndrome of wind-heat hurting collaterals and so on. Blood stasis blocks collaterals, abdominal pain and joint pain, blood stasis blocks renal collaterals, urine and urine, proteinuria can be seen in renal sealing function and loss of function, and the course of pathogenesis is lingering, which suggests that blood stasis blocks collaterals can be indispensable to the pathogenesis of HSPN [103]. However, it is not enough to grasp the syndrome dynamics of children HSPN and the clinical physicians is not sufficient in the relationship between the syndromes and impact of some factors. Therefore, to some extent, the study on the children HSPN has its limitations.

At the present stage, since the pathogenesis of children HSPN has not been clearly understood, there are various kinds of drugs for clinical treatment of children HSPN, and there is insufficient in uniform standards for clinical treatment and the treatment effect is also different. Clinical medical workers have been constantly exploring and studying [104]. However, there is no recognized standard of treatment both domestically and overseas. In many oversea countries, medical researchers advocated clinical observation following up to the child with light illness but there is no consensus in China. They assume that Hormone combined with Immunosuppressor should be used. It is reported that the total effective rate of Cyclosporine A and hormone therapy for children HSPN is $97.4 \%$ [105]. Numerous studies manifested that Glucocorticoid has anti-inflammatory and anti-allergic effects are one of the first choice drugs for clinical treatment of children HSPN. However, clinical observation shows that the effect of glucocorticoid alone is limited and can easily lead to a variety of adverse reactions, such as hormone resistance, hormone dependence and so on. Therefore, the discussion of new therapeutic regimen can be crucial to the treatment of children HSPN. Along with the continuous inheritance and development of Traditional Chinese Medicine, scholars tried best to apply Traditional Chinese Medicine in the clinical treatment of children HSPN. 
Individualized drug used according to TCM syndrome differentiation improves the therapeutic efficiency of children's HSPN to some extent. At present, the treatment of children HSPN is mainly based on the combination of Chinese and Western medicine in China, which is a kind of mature treatment. At present, they apply western medicine to rapidly control the deterioration of the condition and then the traditional Chinese Medicine is to treat in a safe and comprehensive way. In a sense, this can be the most safe and effective way to treat school-age children HSPN.

According to the above studies, domestic scholars have adopted RCT design in the clinical research on the combined treatment for children HSPN to be treated with traditional Chinese medicine and Western medicine. However, the implementation of operation process and matters needing attention were not rigorous enough so that there is a high possibility of implementation bias, which is also a serious problem confronted by the application of traditional Chinese medicine in clinical practice. Therefore, the research should attach great importance to the implementation of the RCT research principle in the future so that it can get more reliable, accurate and instructive data for clinical practice. What's more important, it can provide reliable evidence-based support for the treatment in children with HSPN and treated with the combination of Chinese and Western medicine to the International market. Finally, it can improve the curative effect of the treatment of diseases and benefit the general patients.

\section{References}

[1] Zaffanello $M$, Brugnara $M$, Franchini $M$, et al. Adjuvant treatments for Henoch-Schönlein purpura nephritis in children: A systematic review [J]. Current Therapeutic Research. 2009; 70 (3): 254-265.

[2] Kiryluk K, Moldoveanu Z, Sanders J T, et al. Aberrant glycosylation of IgA1 is inherited in both pediatric $\operatorname{IgA}$ nephropathy and Henoch-Schonlein purpura nephritis [J]. Kidney Int. 2011; 80 (1): 79-87.

[3] Rigante D, Castellazzi L, Bosco A, et al. Is there a crossroad between infections, genetics, and Henoch-Schönlein purpura? [J]. Autoimmunity Reviews. 2013; 12 (10): 1016-1021.

[4] Wang X, Zhang L, Wang Y, et al. Gut microbiota dysbiosis is associated with Henoch-Schönlein Purpura in children [J]. International Immunopharmacology. 2018; 58: 1-8.

[5] Dandan D H Y X. Effects of Chinese herbs in children with Henoch-Schonlein purpura nephritis: a randomized controlled trial [J]. JTCM 2014; 34 (1): 15-22.

[6] Davin J C. Henoch-Schonlein purpura nephritis: pathophysiology, treatment, and future strategy $[\mathrm{J}]$. Clin J Am Soc Nephrol. 2011; 6 (3): 679-689.

[7] Xie G Q, Wen C P, Fan Y S. Progress in study on synergism and detoxification of Chinese medicine for glucocorticoid [J]. J Tradit Chin Med. 2011; 31 (3): 163-168.

[8] Zhou YH, Dong XM. TCM syndrome differentiation rule of purpura nephritis in children based on consensus method [J]. Chinese Medical Guide, 2011; 08: 128-129.
[9] Yi ZW. Interpretation of evidence-based guidelines for the diagnosis and treatment of purpura nephritis in children [J]. Pediatrics in Combination with Chinese and western medicine, 2010; 04: 289-291.

[10] $\mathrm{Lu} \mathrm{XD,} \mathrm{Wu} \mathrm{S,} \mathrm{Fu} \mathrm{SD.} \mathrm{Effects} \mathrm{of} \mathrm{invigorating} \mathrm{spleen} \mathrm{and} \mathrm{cooling}$ blood and activating blood on hemorheology and lymphocyte immunity in children with purpura nephritis [J]. Liaoning journal of traditional Chinese medicine. 2019; 04: 745-748.

[11] Yi HL. Effect of huoxue huayu decoction on infantile purpura nephritis [J]. Journal of shanxi university of traditional Chinese medicine. 2019; 02: 108-110.

[12] Qin YF, li GP. Clinical observation on treating children with purpura nephritis with blood heat and blood stasis with modified xijiao dihuang decoction [J]. Chinese journal of experimental dosage. 2019; 02: 144-149.

[13] Niu DC. Clinical study of tripterygium wilfordii polyglycoside combined with high-dose methylprednisolone intravenous shock in 46 children with allergic purpura nephritis [J]. Northern pharmacy. 201; 01: 62-63.

[14] Zhu W. Therapeutic effect of tripterygium wilfordii polyglycoside on children with purpura nephritis and its effect on immune function [J]. Guizhou medicine. 2019; 03: 448-449.

[15] Ma L, Yuan XF, Li XY, et al. Clinical observation of qingre liangxue huayu decoction in the treatment of infantile purpura nephritis [J]. Chinese medicine guide. 2019; 18: 168-169.

[16] Huo LJ, Wang K, Bai JX, et al. Clinical efficacy and safety analysis of shenyan kangfu tablet combined with hormone in the treatment of children with purpura nephritis [J]. Medical research and education. 2019; 02: 28-32.

[17] Ding LJ. Clinical effect of qingre liangxue jiedu prescription on infantile allergic purpura nephritis and its effect on coagulation mechanism [J]. Chinese journal of pediatrics. 2019; 02: 60-63.

[18] Li Y, Jie SY, huang Y. Effects of bailing capsule therapy on chemochemic protein-1, microalbuminuria and $24 \mathrm{~h}$ urine protein quantification in children with purpura nephritis $[\mathrm{J}]$. Medical equipment. 2018; 13: 97-98.

[19] Huang GC, Sun YN, Lu GN. Effect of tripterygium wilfordii polyglycoside combined with qingre zhixue fang and xiangdan injection in the treatment of children with purpura nephritis [J]. Journal of integrated traditional Chinese and western medicine, 2018, 27; 5: 527-529.

[20] Liu XS, Da CS. Clinical effect of shipi decoction combined with western medicine in the treatment of purpura nephritis in children [J]. Clinical medicine research and Practice. 2018 (18): 129-130.

[21] Wu H J. Effect and side effects of glucocorticoid combined with tripterygium wilfordii polyglycoside on purpura nephritis [J]. Huaihai med. 2018 (03): 354-356.

[22] Huang XH, Hu B. Therapeutic effect of yupingfeng granules combined with double impact therapy on children with severe purpura nephritis $[\mathrm{J}]$. Contemporary medicine. 2018; 21: 73-75.

[23] Yang QY, Lai ZW, Liu GF. Clinical efficacy of tripterygium wilfordii polyglycoside (TWP) in the treatment of infantile purpura nephritis (HSPN) and its effect on immune function in children [J]. Contemporary medicine. 2017; 29: 40-42. 
[24] Wang HN. Effect of tripterygium wilfordii polyglycoside on purpura nephritis [J]. Electronic journal of clinical medicine. 2017; 81: 15986-15988.

[25] Zhang L. Clinical analysis of glucocorticoid combined with tripterygium wilfordii glycoside in the treatment of infantile purpura nephritis $[\mathrm{J}]$. Medical theory and practice. 2017; 02: 243-245.

[26] Liu DF. Clinical study of glucocorticoid combined with tripterygium wilfordii polyglycoside in the treatment of children with purpura nephritis [J]. North China pharmaceutical science. 2017; 07: 74-75.

[27] Liu XG, Shao Q, Liu P. Effects of bailing capsule adjuvant therapy on urinary $\mathrm{McP}-1, \mathrm{mAlb}$ and $24 \mathrm{~h}$ urine protein in children with purpura nephritis [J]. Chinese pharmacists. 2016; 07: 1305-1307.

[28] Li J. Clinical observation of low molecular weight heparin combined with tripterygium wilfordii in the treatment of children with allergic purpura nephritis [J]. Henan medical research. 2016; 04: 719-720.

[29] Sun ZH. Clinical study of fushen decoction in the treatment of children with allergic purpura nephritis [J]. Contemporary medicine. 2016; 14: 156-157.

[30] Zhao QW. Effect of huangkui capsule on children with purpura nephritis [J]. Chinese medical guide. 2016; 13: 185-186.

[31] He JM. Clinical observation on the treatment of 36 cases of allergic purpura nephritis in children with blood-heat blocking collatulosus combined with western medicine $[\mathrm{J}]$. Journal of Pediatrics of Traditional Chinese Medicine. 2016; 05: 52-55.

[32] Gu MM. Effect of liangxue xiaobian granule combined with pidomod on the treatment of wind-heat wound collateral-type allergic purpura nephritis in children and its effect on immune function [J]. Modern journal of integrated Chinese and western medicine. 2016; 32: 3552-3554

[33] Liu XY. To investigate the clinical efficacy and safety of tripterygium wilfordii polyglycoside combined with glucocorticoid in the treatment of children with purpura nephritis [J]. World latest medical information digest. 2016; 58: 80-83.

[34] Yin HW, Wang K. Clinical analysis of glucocorticoid combined with tripterygium wilfordii in the treatment of children with purpura nephritis [J]. Medical BBS. 2016; 10: 141-142.

[35] Zhang XP. Xiaodian empirical prescription for treating purple blemish nephritis in children with blood heat and blood stasis [D]. Anhui university of traditional Chinese medicine, 2016.

[36] Liu FJ. Treatment analysis of children with nephrotic syndrome purpura nephritis with integrated Chinese and western medicine [J]. Journal of chronic diseases. 2015, 16 (05): 524-527

[37] $\mathrm{Lu} \mathrm{W}$. Effect of huaiqi huang granules on serum cytokines in children with allergic purpura nephritis [J]. China pharmaceutical industry. 2015; 18: 47-49.

[38] Zhang SS, Zhang Y. Clinical observation of tripterygium wilfordii polyglycoside and prednisone combined with traditional Chinese medicine in the treatment of children with allergic purpura nephritis [J]. Journal of armed police logistics college (medical edition). 2015; 05: 389-390.
[39] Sun ZM, Wang H, Wei W, et al. Effects of tripterygium wilfordii polyglycoside combined with benapril and heparin on urine protein and urine erythrocyte level in children with allergic purpura nephritis [J]. Chinese modern doctor. 2015; 28: 69-71.

[40] Zhao F, Liu W. Clinical observation of tripterygium wilfordii polyglycoside combined with glucocorticoid in the treatment of children with purpura nephritis [J]. Chinese journal of integrated traditional and western medicine nephropathy. 2015; 09: 822-823.

[41] Liu BZ. Taohong siwu decoction for treatment of purpura nephritis clinical analysis $[\mathrm{J}]$. World latest medical information digest. 2015; 76: 83-84.

[42] Wu HM. Effect of danshen ligustrazine injection on children with purpura nephritis $[\mathrm{J}]$. Contemporary medical review. 2015; 14: $269-270$.

[43] Lin SX. Therapeutic effect of integrated Chinese and western medicine on children with purpura nephritis [J]. Journal of integrated Chinese and western medicine cardiovascular diseases electronic. 2015; 09: 34-35.

[44] Mao GR. Treatment of 32 children with allergic purpura nephritis from spleen $[\mathrm{J}]$. Zhejiang journal of traditional Chinese medicine. 2014; 06: 440.

[45] Guo WG. Effect of danshen ligustrazine on serum endothelin-1 in children with purpura nephritis [J]. Guangming traditional Chinese medicine. 2014; 04: 785-786.

[46] Qiu XY. Clinical analysis of low molecular weight heparin and compound danshen injection in the treatment of children with allergic purpura nephritis [J]. Medical review. 2014; 12: 2269-2271.

[47] Peng YH, Deng LL. Effect of huaiqi huang granules on the treatment of allergic purpura nephritis and its effect on cytokines in serum [J]. Journal of community medicine. 2014; 20: $10-12$

[48] Xiang M. Effects of glucocorticoid and tripterygium wilfordii on children with purpura nephritis [J]. J J med. 2014; 17: 25-27.

[49] Li BN. Efficacy analysis of tripterygium wilfordii polyglycoside in the treatment of children with allergic purpura nephritis [J]. Clinical medicine. 2014; 01: 122.

[50] Li P, HuangFu CR, Tang CH. Therapeutic effect of tripterygium wilfordii polyglycoside on children with purpura nephritis and its effect on immune function [J]. Chinese journal of clinical pharmacology. 2014; 10: 895-897.

[51] Wang P. Clinical observation of xiaobanshenfu decoction in the treatment of hypersensitive purpura nephritis in children with blood-heat injury [D]. Heilongjiang academy of traditional Chinese medicine, 2014.

[52] $\mathrm{Wu} \mathrm{H}$, Chen XH, Zhou S, et al. Efficacy and safety evaluation of low-dose tripterygium wilfordii polyglycoside combined with glucocorticoid in the treatment of children with purpura nephritis $[\mathrm{J}]$. Chinese rural health service management. 2014; 01: 104-107.

[53] Wang J, Diao LL, Liu L. Clinical observation on treating purpura nephritis with yishen jianpi clearing damp huoxue decoction [J]. Chinese medicine clinical journal. 2014; 04: 379-381. 
[54] $\mathrm{Wu} \mathrm{YH.} \mathrm{Treatment} \mathrm{of} \mathrm{children} \mathrm{with} \mathrm{purpura} \mathrm{nephritis} \mathrm{with}$ integrated Chinese and western medicine [J]. Everybody's health (academic edition). 2014; 24: 52-53.

[55] Han DX. Clinical observation of 38 cases of children with allergic purpura nephritis treated by integrated Chinese and western medicine [J]. Chinese folk medicine. 2014; 22: 42-44.

[56] Gong BX. Therapeutic effect of integrated Chinese and western medicine on children with allergic purpura nephritis $[\mathrm{J}]$. Modern journal of integrated Chinese and western medicine. 2014; 14: 1557-1558.

[57] Cheng L. Treatment of children with purpura nephritis with integrated Chinese and western medicine: 38 cases [J]. Modern distance education of Chinese medicine. 2014; 10: 60-69.

[58] Liu YZ. Therapeutic effect of integrated Chinese and western medicine on children with purpura nephritis $[\mathrm{J}]$. Clinical medicine. 2014; 05: 121-123.

[59] Shi ZM. Clinical effect of integrated Chinese and western medicine on children with purpura nephritis [J]. Asia-pacific traditional medicine. $2014 ; 16: 52-53$.

[60] Zhao YX, Guo JY. Treating 32 cases of purpura nephritis with invigorating spleen, invigorating qi, cooling blood and activating blood formula combined with western medicine [J]. Shaanxi journal of traditional Chinese medicine. 2013; 11: 1495-1496.

[61] Huang T. Tanshinone II A sulfonate with glucocorticoid therapy of children with purpura nephritis clinical observation $[\mathrm{J}]$. Journal of contemporary Chinese medicine. 2013; 28: 80-81.

[62] He GX. Effect of kangmin xiaopan decoction combined with western medicine in the treatment of allergic purpura $[\mathrm{J}]$. China practical medicine. 2013; 21: 165-166.

[63] Zeng LL. Efficacy of tripterygium wilfordii polyglycoside combined with hormone in the treatment of children with purpura nephritis and its effect on coagulation function [J]. Journal of liaoning university of traditional Chinese medicine. 2013; 06: 182-183.

[64] Yan HQ, Zeng J. Clinical observation of tripterygium wilfordii polyglycoside in the treatment of children with purpura nephritis [J]. Chinese contemporary medicine. 2013; 28: 86-87.

[65] Jiang JP, Ge DF. Curative effect of qingrezhuyu decoction combined with western medicine in the treatment of 34 cases of purpura nephritis [J]. Chinese journal of acute medicine. 2013; 12: $2133-2134$.

[66] Deng BZ. Xiaodian fang treatment of 32 cases of allergic purpura nephritis $[\mathrm{J}]$. Journal of practical Chinese medicine. 2013; 11: 928.

[67] Li MY. Treatment of children with purpura nephritis by integrated Chinese and western medicine [J]. China health industry. 2013; 21: 123-125.

[68] Jin JW, Wang R. Clinical observation of yuduqing combined with western medicine in the treatment of children with allergic purpura nephritis: 42 cases [J]. Hebei journal of traditional Chinese medicine. 2013; 09: 1410-1416.

[69] Yuan PZ. Clinical analysis of 35 cases of children with purpura nephritis treated by integrated Chinese and western medicine [J]. Chinese and foreign medical research. 2013; 07: 46-47.
[70] Wang YJ, Zhang LM, Men GG, et al. Clinical observation of tripterygium wilfordii polyglycoside combined with hormone in the treatment of children with purpura nephritis [J]. Journal of pediatric pharmacy. 2012; 06: 24-26.

[71] Ji RW. Treatment of 35 cases of allergic purpura nephritis with integrated Chinese and western medicine [J]. Inner Mongolia journal of traditional Chinese medicine. 2012; 04: 27-28.

[72] Li YE, ZhangTD. Clinical observation on syndrome differentiation in treatment of purpura nephritis [J]. Sichuan journal of traditional Chinese medicine. 2011; 02: 87.

[73] Chen CL. Efficacy of low molecular weight heparin combined with compound danshen injection in treating children with allergic purpura nephritis [J]. Medical information. 2011; 09: 4886-4887.

[74] Pang FM. Effect of tripterygium wilfordii polyglycoside on treatment of 34 cases of purpura nephritis [J]. Hainan med. 2011; 10: 84-85.

[75] Pei HB, Ji HP. 37 cases of children with allergic purpura nephritis treated by shengyang sanhuo decoction combined with western medicine [J]. Journal of practical Chinese medicine internal medicine. 2011; 08: 61-62.

[76] Wang CF, Xia Y, Liu JC. Therapeutic effect of yishen granules on children with allergic purpura nephritis [J]. Guangming traditional Chinese medicine. 2011; 06: 1143-1144.

[77] Guo YH, Jin D. 30 cases of children with purpura nephritis treated by integrated Chinese and western medicine $[\mathrm{J}]$. Western Chinese medicine. 2011; 10: 71-73.

[78] Li JC, Gao YX. Therapeutic effect of integrated Chinese and western medicine on 30 cases of allergic purpura [J]. Shanxi journal of medicine. 2011; 10: 1009-1010.

[79] Zhao ZY, Qi YF. Therapeutic effect of integrated Chinese and western medicine on children with purpura nephritis: 40 cases $[\mathrm{J}]$. Pediatrics of integrated Chinese and western medicine, 2011; 02: 161-162.

[80] Peng HP. Treatment of 50 cases of allergic purpura nephritis with integrated Chinese and western medicine $[\mathrm{J}]$. Guangming traditional Chinese medicine. 2011; 06: 1213-1214.

[81] Zhao YD. Clinical analysis and study of integrated Chinese and western medicine in the treatment of children with allergic purpura nephritis [D]. Lanzhou university, 2011.

[82] Hao JJ. Treatment of children with nephrotic syndrome purpura nephritis with integrated Chinese and western medicine $[\mathrm{J}]$. Chinese and foreign medical treatment. 2011; 01: 53-55.

[83] Lu J, Liu X. Treatment of infantile purpura nephritis with integrated Chinese and western medicine [J]. World journal of integrated Chinese and western medicine. 2010; 04: 351.

[84] Chen ZH. Summary of 38 cases of children with purpura nephritis treated by integrated Chinese and western medicine [J]. Hunan journal of traditional Chinese medicine. 2010; 03: 3-5.

[85] Yu FM. Clinical observation on 28 cases of purpura nephritis treated by integrated Chinese and western medicine $[\mathrm{J}]$. Chinese community physician. 2010; 08: 116.

[86] Zhang TD, Li YF. Treatment of 30 cases of purpura nephritis with integrated Chinese and western medicine [J]. Chinese journal of acute medicine. 2010; 04: 667-668. 
[87] Guo QY, Ren XQ. Syndrome differentiation and treatment combined with prednisone for children with nephrotic syndrome purpura nephritis: 42 cases [J]. Shaanxi journal of traditional Chinese medicine. 2009; 07: 798-799.

[88] Huang QY. Efficacy of low molecular weight heparin combined with tripterygium wilfordii glycoside in the treatment of children with allergic purpura nephritis [J]. Chinese and foreign medical treatment. 2009; 07: 72.

[89] Chen CB, Wang M, Lu Wei. Effect of cyclophosphamide injection combined with prednisone on children with purpura nephritis [J]. Chinese journal of nephropathy of integrated traditional and western medicine. 2009; 11: 1001-1002.

[90] Ma XC, Wang LL, Feng JH. Clinical observation of shenyankang in the treatment of children with allergic purpura nephritis [J]. Jilin traditional Chinese medicine. 2009; 10: 845-846.

[91] Li G, Zhang BQ, Liu ZG. The advantages of combining Chinese and western medicine in the treatment of children with purpura nephritis $[\mathrm{J}]$. Chinese and foreign medical treatment. 2009; 03: 82 .

[92] Huang WY. Clinical observation of 35 cases of children with purpura nephritis treated by integrated Chinese and western medicine [J]. Sichuan journal of traditional Chinese medicine. 2009; 06: 99-100.

[93] Wu ZC, Zhang Li. Treatment of children with allergic purpura by integrated Chinese and western medicine: 92 cases [J]. Chinese folk medicine. 2009; 14: 70.

[94] Wang J. Treatment of children with purpura nephritis by integrated Chinese and western medicine [J]. Journal of practical Chinese medicine. 2009; 06: 381-382.

[95] Wang JL, Luo GX. clinical observation of jiedu huayu decoction in the treatment of allergic purpura nephritis [J]. Chinese journal of traditional Chinese medicine. 2007; 12: 2655-2656.

[96] Wang YL, Zhang LM, Xin RZ, et al. Clinical observation of tripterygium wilfordii glycosides in treating children with allergic purpura nephritis $[\mathrm{J}]$. Chinese journal of coal industry medicine. 2007; 02: 161-162.

[97] Fan WW, Jiang XB, Shi ZX, et al. Treatment of children with allergic purpura nephritis by integrated traditional Chinese and western medicine $[\mathrm{J}]$. Hunan journal of traditional Chinese medicine. 2007; 02: 28-30.

[98] Zhang JP, Xie FP, Long YC. tripterygium wilfordii glycosides in the treatment of children with allergic purpura nephritis [J]. Clinical research. 2006; 06: 952-953.

[99] Chang WJ, Fang JA. Clinical study of yiqitonglin capsule in the treatment of 60 cases of allergic purpura nephritis [J]. Chinese journal of integrated traditional and western medicine nephropathy. 2006; 08: 424-425.

[100] Song MS. Xiere xiaopan decoction in the treatment of infantile purpura nephritis $[\mathrm{J}]$. Chinese journal of nephropathy, 2005; 12: 727.

[101] Mao YY, Wang HP, Zhou QF. Therapeutic effect of integrated Chinese and western medicine on children with purpura nephritis $[\mathrm{J}]$. Journal of shandong university of traditional Chinese medicine. 2004; 01: 41-43.

[102] Zhang Y, Li A, Wen J, et al. Kidney Injury Molecule-1 Level is Associated with the Severity of Renal Interstitial Injury and Prognosis in Adult Henoch-Schönlein Purpura Nephritis [J]. Archives of Medical Research. 2017; 48 (5): 449-458.

[103] Sun J. Progress of traditional Chinese medicine treatment of purpura kidney (HSPN) in children [J]. Jilin traditional Chinese medicine (Chin) 2007; 05: 68-69.

[104] Chen Q, Wu YQ, Li N, et al. Efficacy and safety evaluation of tripterygium wilfordii in the treatment of purpura nephritis in children and meta-analysis [Z]. Nanning, guangxi, China (Chin) $2015 ; 1$.

[105] Chen Y, Yu L, Zhang Y. Efficacy analysis of cyclosporine A combined with hormone in the treatment of purpura nephritis in children $[\mathrm{J}]$. Journal of clinical and experimental medicine (Chin) 2010; 17: 1294-1295. 